\title{
Dynamical response of a rocking rigid block
}

Y. Liu, ${ }^{1, a)}$ J. Páez Chávez, ${ }^{2,3}$ P. Brzeski, ${ }^{4}$ and P. Perlikowski ${ }^{4}$

${ }^{1)}$ College of Engineering, Mathematics and Physical Sciences, University of Exeter, North Park Road, Exeter, EX4 4QF, UK.

${ }^{2)}$ Center for Applied Dynamical Systems and Computational Methods (CADSCOM), Faculty of Natural Sciences and Mathematics, Escuela Superior Politécnica del Litoral, P.O. Box 09-01-5863, Guayaquil, Ecuador.

${ }^{3)}$ Center for Dynamics, Department of Mathematics, TU Dresden, D-01062 Dresden, Germany.

4) Division of Dynamics, Lodz University of Technology, Stefanowskiego 1/15, 90-924 Lodz, Poland.

(Dated: 19 June 2021)

This paper investigates the complex dynamical behavior of a rigid block structure under harmonic ground excitation, thereby mimicking, for instance, the oscillation of the system under seismic excitation or containers placed on a ship under periodic acting of sea waves. The equations of motion are derived assuming a large frictional coefficient at the interface between the block and the ground, in such a way that sliding cannot occur. In addition, the mathematical model assumes a loss of kinetic energy when an impact with the ground takes place. The resulting mathematical model is then formulated and studied in the framework of impulsive dynamical systems. Its complex dynamical response is studied in detail using two different approaches, based on direct numerical integration and path-following techniques, the latter implemented via the continuation platform $\mathrm{COCO}$ (Dankowicz \& Schilder). Our study reveals the presence of various dynamical phenomena, such as branching points, fold and period-doubling bifurcation of limit cycles, symmetric and asymmetric periodic responses, as well as chaotic motion. By using basin stability method we also investigate the properties of solutions and their ranges of existence in phase and parameters spaces. Moreover, the study considers ground excitation conditions leading to the overturning of the block structure and shows parameters regions wherein such behavior can be avoided.

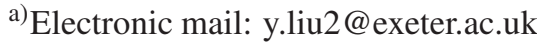


The dynamics of the rocking block has been studied for more than 60th years, and still, we can find new inspiring, complex phenomena in such a simple system. Numerous structures are modeled as rocking blocks, i.e., tall buildings, free-standing power transformers, cell towers, silos, and containers. The rocking block is used to mimic the slander structure, which is not or badly connected to the ground. Hence, the transversal movements of the foundation can lead to the appearance of the so-called rocking motion. The rocking motion will cause an overturn or structure's damage for some sets of the system's excitation parameters. We distinguish two main types of excitation. The first one is an earthquake, and it affects the system placed on the ground. In contrast, the second one is the sea wave acting on the container ships. As it is easy to see, both of them have significantly different properties. The earthquake has a relatively high and broad frequency spectrum, and it didn't last long. Hence, it affects the structure in a short time with a very harsh forcing. Usually, it results in damage or overturns of the system. On the other hand, the sea wave has a lower frequency, lasts for a long time and varies slowly. Therefore, in addition to the damage or overturn, we can observe a stable oscillatory motion. This paper focuses on the second type of excitation, and we model it as a periodic function acting on the rigid block. Of course, in extreme events, the sea wave excitation has similar or worst properties as the earthquake, and one should study such cases assuming forcing with the complex signal. The rocking motion is modeled as rotations with respect to the structure's left and right bottom corner. The impacts occur during the motion, so the model is not only nonlinear but also piecewise. Due to discontinuity, we observe various dynamical phenomena, including bifurcations and the coexistence of solutions. We describe the bifurcation scenario leading from steady-state to chaos via grazing, fold, symmetry breaking, and period-doubling bifurcations. We show the bifurcation borders in two parameters space, i.e., the amplitude and frequency of the excitation. Such projection allows presenting ranges where the given solution is stable and its destabilization scenario. In the considered system, we always observe the coexistence of at least two solutions (overturn and equilibrium/periodic/chaotic). To reveal the structure of the multistable phase space, we investigate the system with sample-based methods. Presented results describe the properties of solutions and their ranges of existence in phase and parameter spaces. We show the probability of the existence of solutions as a function of initial conditions and parameter values. Hence, we can see the influence of those quantities on the appearance of solutions and define the ranges where the given state is the most or the less 
probable.

\section{INTRODUCTION}

The rocking response of slender rigid structures due to the excitation at its base, e.g. during an earthquake, has been the subject of great interest for many researchers, see 1-3. Such a rocking motion in large engineering structures, such as buildings, free standing power transformers and cell towers can cause catastrophic failures as a consequence of overturning, both from an economical and human perspective. One of the first systematic studies in this area was pioneered by Housner ${ }^{4}$ who has established a rocking block model that has been widely used by many researchers in the past. His study suggests that the survival of a tall slender structure during earthquakes depends on a scale effect making the structure more stable against overturning. The block equations of motion were described by piecewise-smooth equations depending on the sign of its rotation angle. Despite its apparent simplicity, the problem poses serious difficulties from an analytical point of view. Therefore, the majority of previous works has employed numerical approaches to analyze the underlying structure, e.g., 5-13, most of which are based on studies relying on direct numerical simulations. Therefore, in the present work we will undertake a comprehensive parametric study of the block structure under ground excitation using specialized numerical techniques, based on a numerical continuation approach.

Past numerical works in this research mainly focused on understanding the fundamental properties of the rocking block model. For example, Yim el al. ${ }^{5}$ studied the rocking response of rigid blocks subjected to earthquake ground motion, and developed a numerical procedure to solve the nonlinear equations of motion governing the rocking motion. They also studied the problem from a probabilistic point of view with the ground motion modelled as a random process. Their numerical results suggest that the response of the block is sensitive to small changes in its size and slenderness ratio, as well as the features of the ground motion. Ishiyama ${ }^{6}$ developed a computer program to simulate the motion of a rigid body subjected to horizontal and vertical ground motions. It was found that the criteria for overturning of the body depended on the horizontal acceleration and the velocity of the ground. Hogan ${ }^{7}$ analyzed a rigid block undergoing harmonic forcing without damping. An in-depth analytical and numerical study has been performed for the slender structures because in this case, it is possible to linearize equations of the motion and find their exact 
solutions. However, such a specific ratio between the rocking block dimensions is not typical for real-world systems and do not give a comprehensive overview of most existing system's dynamics. Hogan presented the stability of resonant periodic orbits in the amplitude-frequency of the excitation plane for different restitution coefficient values. The values of these parameters significantly influence ranges of the resonant periodic solutions' existence. Hence, its proper selection is crucial for the correctness of modeling of the system's behavior. Then, the Author presented the numerical study of the nonlinear equations of motion for slander and non-slender rocking blocks. He showed the asymmetric solutions and period-doubling sequence leading to chaos. Then, we can find one basin of attractions with fractal properties, which confirms that multiple solutions coexist in this system. Finally, we see the analysis of the system response to a short time excitation which simulate areal earthquake. Hogan assumed that an earthquake has a finite time of duration, and based on such study, one can predict the conditions leading to non-toppling or toppling states. The following paper by Hogan ${ }^{14}$ extended the above-described results and focused on the coexistence of resonant periodic solutions in the system. He proved that for adjacent initial conditions, one can observe transitions to different solutions, hence the system is multistable. At the end of the paper, the comparison of theoretical results with experiments by Tso and Wong ${ }^{15,16}$ is presented. The matching of results is satisfactory, and simulations help to explain experimental observations. Hogan also investigated the effect of damping on rigid block motion under harmonic forcing in 9. The study is analytical and bases on linearized equations of the motion, hence it assumes a slender rigid block. The Author showed several two parameters plots showing the stability of resonant periodic solutions for four parameters of the system, i.e., the amplitude and frequency of the forcing, restitution and damping coefficients. Then, he supported his study with numerical computation of the nonlinear equations of the motion and showed the multiple solutions, asymmetric responses, period- and impact-doubling cascades. This study shows that the rocking block under harmonic excitation has complex dynamics, and a plethora of different behavior can occur. It is a motivation to study the system with new numerical tools to have a more general overview of dynamics, the structure of the phase space and bifurcation scenarios.

Lenci and Rega ${ }^{17}$ developed a method to eliminate the heteroclinic intersections embedded in the block's dynamics by controlling the periodic excitation. In 10, a comprehensive experimental investigation was carried out to study the rocking response of four blue granite stones with different geometrical characteristics under free, harmonic, and random vibrations. In particular, two simulation approaches, one through Lagrangian formalism and the second one employing discrete 
element model, were used to identify the models' parameters and study the rigid-block structure's dynamic response. In 11, Dimitrakopoulos and DeJong introduced new closed-form solutions and original similarity laws which can shed light on the fundamental aspects of the rocking block. The focus was on the transient dynamics of the rocking block under finite-duration excitations. In 12 , closed-form expressions were obtained for the dynamic response of a rocking block, whereas rigorous overturning criteria were established for conditions with and without impact. Later on, Brzeski et al. ${ }^{13}$ studied the influence of tuned mass absorbers on the dynamics of rigid block in order to prevent overturning. The presented method was used to optimise tuned mass absorber's parameters to achieve the highest chance that the block will remain standing. Final results confirmed that it was possible to design and tune the stabilising device in such a way that it was highly effective regardless of the rigid block asymmetry. The influence of the excitation's initial phase on the response of the structure has been studied for four selected values of the phase by Lenci and Rega $^{18}$. The authors show how the dynamics of the system varies and which phase shift is the best for stabilization of the rocking block. While in 19 the response of the rigid block on sine or cosine impulse is presented. Moreover, the authors show the analysis of values of initial conditions on the response of the structure. However, the selection of possible values of initial conditions is limited to values close to zero.

This paper presents a detailed numerical study of the dynamical response of the rocking block model with a special focus on its symmetric and asymmetric solutions. We are particularly interested in the periodic solutions of the system and the evolution of such responses under the changing parameters of base excitation. When non-smooth nonlinearity, i.e. impacts in this system, is involved, the dynamical study of systems of this type (see 20-22) brings well-known challenges from a numerical point of view. In order to study the behavior of the rocking block, we will adopt two different types of numerical approaches, namely, direct numerical integration and path-following methods for non-smooth dynamical systems ${ }^{23}$. For this kind of systems, the state space can be divided into disjoint subregions, so the rocking block model in each region can be described by a smooth vector field. Hence, a special care needs to be considered in order to get reliable numerical approximations of the system response in an efficient way ${ }^{24}$.

Path-following (continuation) methods are well-established techniques in applied mathematics ${ }^{25}$, which allow a systematic analysis of a system response under the variation of control parameters. For piecewise-smooth systems, specialized computational tools for continuation, such as SlideCont ${ }^{26}$, TC-HAT $^{27}$ and $\mathrm{COCO}^{28}$, have been developed, and the latter will be adopted in the 
current work for the numerical study of the rocking block model. The MATLAB-based numerical platform COCO supports the continuation and bifurcation detection of multi-segment periodic orbits, a feature that has been extensively used in the past for the numerical bifurcation study of piecewise-smooth dynamical systems. For example, it was used to study the robustness of a controller for controlling the multistability in a vibro-impact capsule system ${ }^{29}$. In 21 , it was adopted to determine the optimal control parameters in an impacting system in terms of its energy expenditure due to the control signal and transient behavior of the control error. Liu et al. ${ }^{30}$ employed the continuation platform COCO to investigate the dynamical response of a higher order drifting oscillator. By using the platform, the authors were able to eliminate the bistability observed in the system and improve system's performance with respect to its rate of penetration. In the current work, we will formulate the rocking block model in the framework of piecewise-smooth dynamical systems and employ the continuation platform COCO to study its complex dynamical response in detail. Our main concern will be to understand the bifurcation structure of the model, which will reveal the presence of branching points, fold and period-doubling bifurcation of limit cycles, symmetric and asymmetric periodic responses, as well as chaotic motion. In addition, one of the main concerns will be to identify ground oscillation modes leading to overturning and how to avoid them by considering suitable parameter regions where such events can be avoided. This work can then be used as starting point for developing effective control strategies to prevent overturning during rocking motion of the rigid block.

The second method applied to study the dynamics of the system considered in this paper is a basin stability method ${ }^{31,32}$. It enables to quantify the stability of solutions based on the probability of reaching given attractor from random initial conditions. To calculate the basin stability measure one has to perform a significant number of Bernoulli trials and classify final solution reached in each trial. The efficiency of the basin stability method in comparison to the basins of attractions is especially visible when the dimension of phase space is larger than two. In such a case, the classical approach requires much larger computational effort and interpretation of results is very complex (necessity of projections of results on two dimensional cross-sections of multi-dimensional phase space $)^{33-35}$. The basin stability method gives statistical information on existing solutions (the volume of basins of attractions in relation to the total volume of considered phase space) and their location in the phase space. Moreover, drawing of inital conditions allows to monitor the probability to reach given solution. We also have more certainty that all existing attractors have been detected and no rare solutions have been overlooked ${ }^{36-39}$. In our previous 
papers $40-42$ we extend the basin stability approach assuming that values of system parameters are also somehow random. It allows the fast scanning of the initial conditions and the parameter spaces to find all meaningful solutions that exist in the system and the ranges of their existence. Thus, we can determine where the system is mono-stable/multi-stable or which perturbation or parameter mismatch can lead to destabilization of the system ${ }^{43}$. The important data can be obtained from two dimensional diagram, which shows the probability of given solution as a function of two variables. Such data is important in practical applications because in many cases parameters of the system are varying or it is impossible to identify their precise values and we must be sure that there is no danger of sudden jump to another solution or appearance of bifurcation ${ }^{44}$. In this paper, we used this method to investigate the structure of the phase space, and identified the regions of multi-stability and the dependence of a given type of solution on the system parameters.

The rest of the paper is organized as follows. In Section 2, the physical model and equations of motion of the rocking block are derived. Section 3 introduces the mathematical formulation of the rocking block model as a piecewise-smooth dynamical system. Then, a detailed numerical investigation using the continuation methods via $\mathrm{COCO}$, including one- and two-parameter studies, is presented in Section 4. In Section 5 we show the solutions coexisting in the system and their probability of occurrence using basin stability method. Finally, some concluding remarks are drawn in Section 6.

\section{PHYSICAL MODEL}

\section{A. Equations of motion}

In the current work the system presented in Fig. 1 is considered, where a rigid block with the mass $M$ stands on the base subjected to periodic excitation with infinite rigidity. Here, we posit only horizontal motion of the base, $\ddot{x}=A \Omega^{2} \sin (\Omega t)$, is considered, so $x=-A \sin (\Omega t)$, and the coefficient of friction between the block and the base is sufficiently large to prevent sliding. Therefore, the block stands still or performs oscillations around one of its corners (i.e. point $O^{+}$or $O^{-}$) that is currently in contact with the ground. The angular position of the block is given by the generalized coordinate $\varphi$, which describes its angular deflection from the standing still position. We assume that the distances between the center of mass and its corners are equal, given by the length $R$. The slenderness of the block is described by $\theta_{b}$, which is the angle between the lower 
edge of the block and the line that connects block's center of mass with its corner. If there is no external excitation, the block will overturn for angles larger than $\frac{\pi}{2}-\theta_{b}$, and we define it as the critical angle for the block.

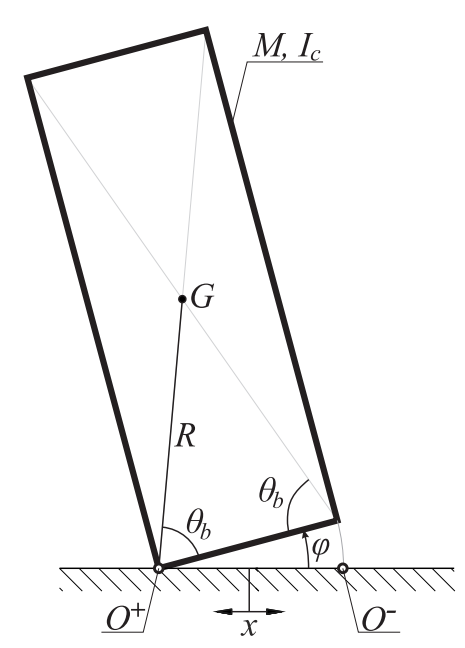

FIG. 1. Model of the rigid rocking block.

When $\varphi>0$, the block is rocking around the point $O^{+}$the equation of motion is given by:

$$
\begin{aligned}
\left(I_{c}+M R^{2}\right) \ddot{\varphi}- & M R \\
& \sin \left(\theta_{b}+\varphi\right) A \Omega^{2} \sin (\Omega t) \\
+ & g M R \cos \left(\theta_{b}+\varphi\right)+c_{b} \dot{\varphi}=0,
\end{aligned}
$$

while for $\varphi<0$,

$$
\begin{aligned}
\left(I_{c}+M R^{2}\right) \ddot{\varphi}- & M R \sin \left(\theta_{b}-\varphi\right) A \Omega^{2} \sin (\Omega t) \\
& -g M R \cos \left(\theta_{b}-\varphi\right)+c_{b} \dot{\varphi}=0,
\end{aligned}
$$

where $c_{b}$ is the viscous damping coefficient in the pivot points of the block, $g$ is the acceleration due to gravity.

The block will start rotation around one of the pivot point $\left(\mathrm{O}^{+}\right.$or $\left.\mathrm{O}^{-}\right)$depending on its acceleration $\ddot{x}$. This motion continues up to the moment when the block overturns or until falls back to the standing position $\varphi=0$ knocking the ground. Here, we assume that there is no bouncing from the block, so after the collision, the rotation of the block continues around one of the pivot points, and the angular momentum of the system is conserved. To model the contact between the block and the ground, the impact law 5 is adopted as

$$
\dot{\varphi}^{+}=e \dot{\varphi}^{-},
$$


where $\dot{\varphi}^{-}$and $\dot{\varphi}^{+}$are the angular velocities of the block just before and after the impact, respectively. The corresponding restitution coefficient $e$ is defined as

$$
e=1-\frac{3}{2} \sin ^{2}\left(\frac{\pi}{2}-\theta_{b}\right)
$$

which is determined from the conservation of angular momentum ${ }^{5}$ and depends on the slenderness of the block $\theta_{b}$ only. It is worth noting that the energy loss due to the contact depends on the material property of the block and the base. However, for the idealized rigid block and base, formula (4) can give a good approximation of the restitution coefficient which has been widely adopted. The mathematical model described above will be investigated for the parameter values given in Table I, which correspond to a realistic physical scenario.

TABLE I. Parameter values for the rocking block model.

\begin{tabular}{|c|c|c|}
\hline Parameters & Values/Range & Units \\
\hline$M$ & $20 \times 10^{3}$ & $\mathrm{~kg}$ \\
\hline$c_{b}$ & 101 & $\mathrm{Nms}$ \\
\hline$g$ & 9.81 & $\mathrm{~m} / \mathrm{s}^{2}$ \\
\hline$R$ & 3.764 & $\mathrm{~m}$ \\
\hline$\theta_{b}$ & 0.8795 & $\mathrm{rad}$ \\
\hline$A$ & $(0,4]$ & $\mathrm{m}$ \\
\hline$\Omega$ & {$\left[\frac{\pi}{4}, 2 \pi\right]$} & $\mathrm{rad} / \mathrm{s}$ \\
\hline
\end{tabular}

For our numerical investigation, it is convenient to write the equations of motion in nondimensional form according to the formulae

$$
\begin{gathered}
\Omega_{0}=\sqrt{\frac{g}{R}}, \quad \tilde{t}=\Omega_{0} t, \quad \omega=\frac{\Omega}{\Omega_{0}}, \\
\xi=\frac{c_{b}}{M R^{2} \Omega_{0}}, \quad \alpha=\frac{A}{R} .
\end{gathered}
$$

By using these transformations, we obtain a set of dimensionless equations for the motion of the rocking block as follows. For $\varphi>0$, we have

$$
\begin{aligned}
\varphi^{\prime \prime}-\frac{3}{4} \alpha \omega^{2} \sin \left(\theta_{b}+\varphi\right) & \sin (\omega \widetilde{t}) \\
& +\frac{3}{4} \cos \left(\theta_{b}+\varphi\right)+\frac{3}{4} \xi \varphi^{\prime}=0,
\end{aligned}
$$


while for $\varphi<0$, it gives

$$
\begin{aligned}
\varphi^{\prime \prime}-\frac{3}{4} \alpha \omega^{2} \sin \left(\theta_{b}-\varphi\right) & \sin (\omega \widetilde{t}) \\
& -\frac{3}{4} \cos \left(\theta_{b}-\varphi\right)+\frac{3}{4} \xi \varphi^{\prime}=0,
\end{aligned}
$$

where the prime symbol denotes differentiation with respect to the non-dimensional time $\widetilde{t}$. In what follows, tildes will be dropped for the sake of simplicity.

\section{DESCRIPTION OF THE MODEL AS A NON-SMOOTH DYNAMICAL SYSTEM}

The rocking block model (3), (6), (7) introduced in the previous section belongs to the class

of non-smooth dynamical systems ${ }^{23}$. Such class of systems naturally arises when the underlying governing laws of a certain problem include non-smooth phenomena, for instance (hard or soft) impacts, friction, switches, etc. The equations of motion in these cases can be written in terms of a piecewise-smooth vector field in combination with a set of jump laws, hence producing discontinuities in the solution and in its first or higher-order derivatives. In the considered rocking block model, a discontinuity in the solution is produced by the restitution law assumed in (3), which accounts for the instantaneous velocity change when the block hits the ground $(\varphi=0)$.

In general, a non-smooth dynamical system can be characterized by three main components: a set of (smooth) vector fields, event functions and jump functions. In this way, a system trajectory is defined by a collection of operation modes whose evolution is described by a specific smooth vector field. The termination condition of a given operation mode is defined in terms of the zeroes of a particular event function ( $\varphi=0$ in our case). Then, the initial point for the next operation mode is determined by a given jump function, which maps the terminal point of the current mode to the initial point of the next one. In our case, the jump function defines the angular velocity of the block just after an impact with the ground occurs, according to the restitution law (3). This formulation enables the application of specialized numerical techniques for the analysis of nonsmooth dynamical systems, for instance via path-following (continuation) methods ${ }^{27,28}$.

For the study of the rocking block model via path-following methods, it is convenient to write the governing equations in autonomous form. To this end, we will employ a self-excited, nonlinear 
oscillator given by 25 :

$$
\left\{\begin{array}{l}
r^{\prime}(t)=r(t)+\omega s(t)-r(t)\left(r(t)^{2}+s(t)^{2}\right), \\
s^{\prime}(t)=s(t)-\omega r(t)-s(t)\left(r(t)^{2}+s(t)^{2}\right)
\end{array}\right.
$$

which possesses the asymptotically stable solution $r(t)=\sin (\omega t), s(t)=\cos (\omega t)$, for all $t \geq 0$. In this way, we can write the periodically forced rocking block model in autonomous form, which then allows us to study the model via numerical continuation methods.
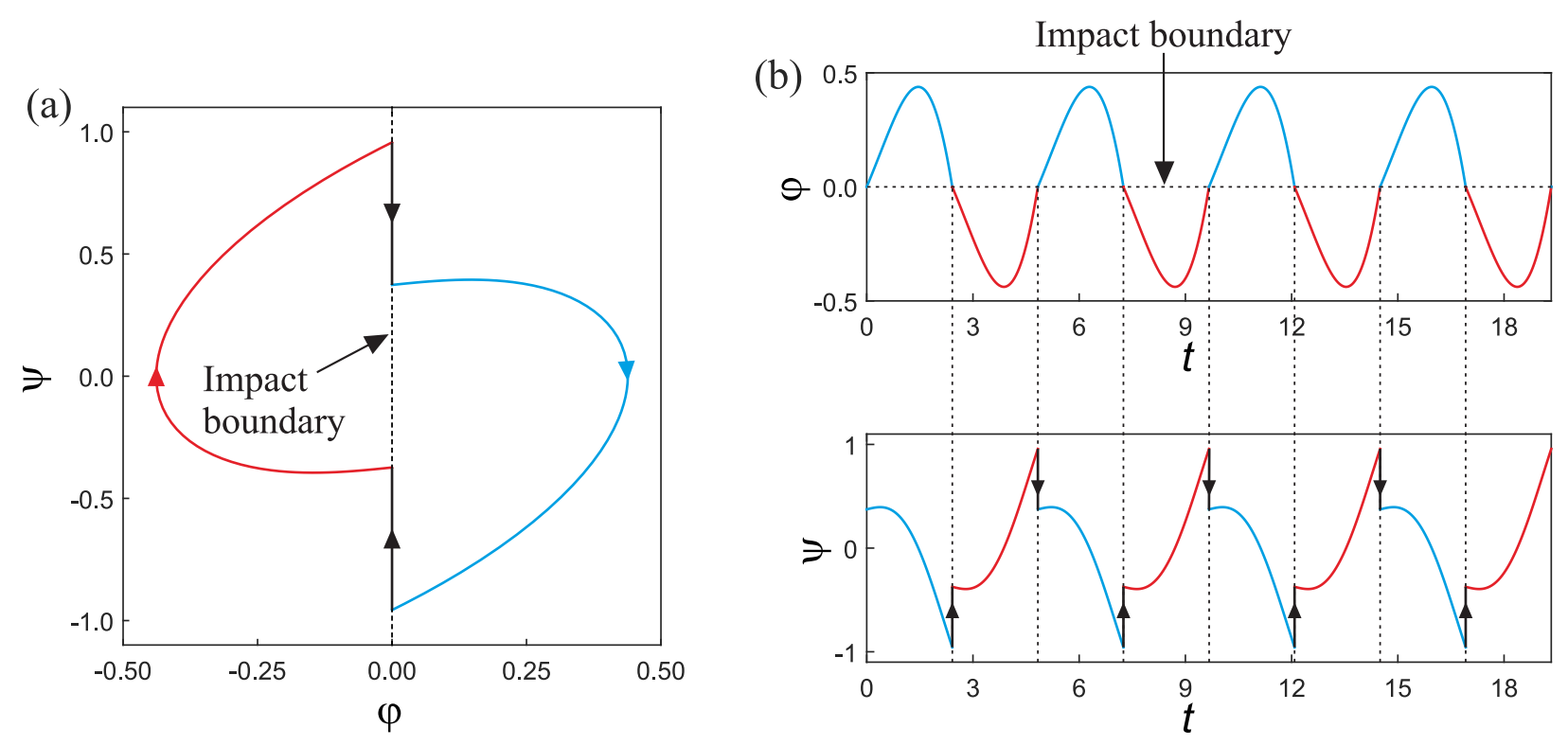

FIG. 2. Periodic response of the rocking block model (9), obtained for the parameter values $\omega=1.3$, $\alpha=0.66, \theta_{b}=0.8795$ and $\xi=0.00022$. (a) Phase portrait of the periodic solution and (b) time series showing the angular position $\varphi$ and angular velocity $\psi$. Here, the impact boundary $\varphi=0$ is depicted as a dotted line. The colors red and blue mark the solution segments for which $\varphi<0$ and $\varphi>0$, respectively. The vertical arrows represent the instantaneous change in the angular velocity when the block hits the ground $(\varphi=0)$.

Let us define $\lambda:=\left(\omega, \alpha, \xi, \theta_{b}\right) \in\left(\mathbb{R}^{+}\right)^{4}$ and $z:=(\varphi, \psi, r, s)^{T} \in\left[-\frac{\pi}{2}+\theta_{b}, \frac{\pi}{2}-\theta_{b}\right] \times \mathbb{R}^{3}$ as the parameters and state variables of the system, respectively, where $\psi$ will capture the angular velocity (see Eqns. (9)-(11) below). In this setting, the rocking block model (3), (6), (7) introduced 
in the previous section can be written as a non-smooth dynamical system as follows

$$
\begin{cases}z^{\prime}(t)=f_{+}(z(t), \lambda), & \varphi(t)>0, \\ z^{\prime}(t)=f_{-}(z(t), \lambda), & \varphi(t)<0, \\ \left(\varphi\left(t^{+}\right), \psi\left(t^{+}\right), r\left(t^{+}\right), s\left(t^{+}\right)\right)=\left(\varphi\left(t^{-}\right), e \psi\left(t^{-}\right), r\left(t^{-}\right), s\left(t^{-}\right)\right), & \varphi(t)=0, \quad \varphi^{\prime}(t) \neq 0\end{cases}
$$

where the vector fields are defined as

$$
\begin{gathered}
f_{+}(z, \lambda):=\left(\begin{array}{c}
\psi \\
\frac{3}{4}\left(\alpha \omega^{2} r \sin \left(\theta_{b}+\varphi\right)-\cos \left(\theta_{b}+\varphi\right)-\xi \psi\right) \\
r+\omega s-r\left(r^{2}+s^{2}\right) \\
s-\omega r-s\left(r^{2}+s^{2}\right)
\end{array}\right), \\
f_{-}(z, \lambda):=\left(\begin{array}{c}
\psi \\
\frac{3}{4}\left(\alpha \omega^{2} r \sin \left(\theta_{b}-\varphi\right)+\cos \left(\theta_{b}-\varphi\right)-\xi \psi\right) \\
r+\omega s-r\left(r^{2}+s^{2}\right) \\
s-\omega r-s\left(r^{2}+s^{2}\right)
\end{array}\right) .
\end{gathered}
$$

In addition, for our numerical investigation we will consider the following solution measure

$$
\text { Amp }:=\max _{0 \leq t \leq T_{0}}|\varphi(t)|
$$

computed for a given $T_{0}$-periodic solution. In this way, we will be able to detect critical points defined by the condition $\varphi=-\frac{\pi}{2}+\theta_{b}$ or $\varphi=\frac{\pi}{2}-\theta_{b}$. They define static boundaries where block overturning can occur. For the numerical implementation, the quantity defined in (12) is computed from the discretized solution, using the max function.

\section{NUMERICAL STUDY OF THE PERIODICALLY EXCITED ROCKING BLOCK}

In this section our main goal will be the numerical investigation of the rocking block motion subject to periodic base excitation, introduced in Section II. As mentioned earlier, the considered physical model may represent a number of practical scenarios, such as buildings, silos, containers, scaffoldings, free standing power transformers, etc., and one of the main questions in this matter is to determine the conditions under which overturning of the object occurs. For the considered physical configuration, we will pay close attention to solutions crossing the boundaries $\varphi=\theta_{b}-\frac{\pi}{2}$ 
(a)

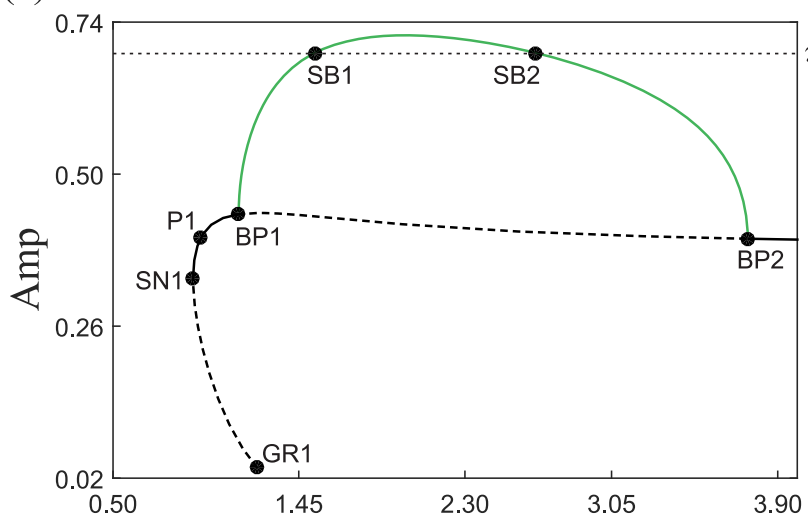

$\omega$

(c)

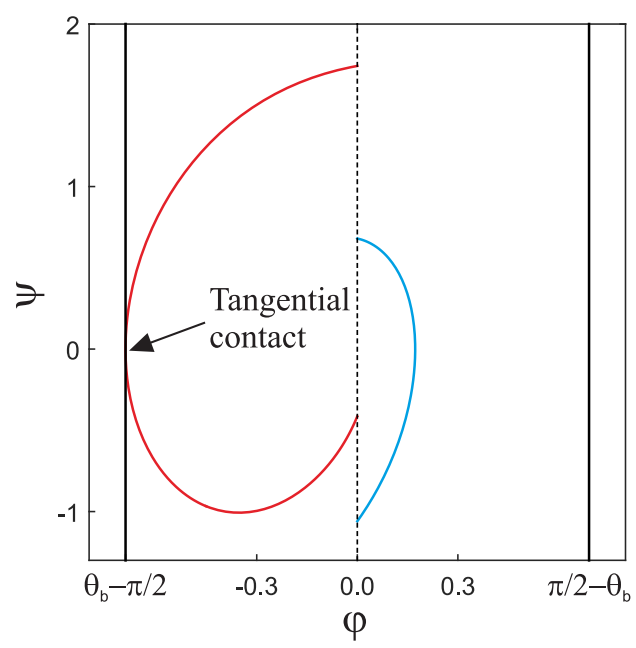

(b)

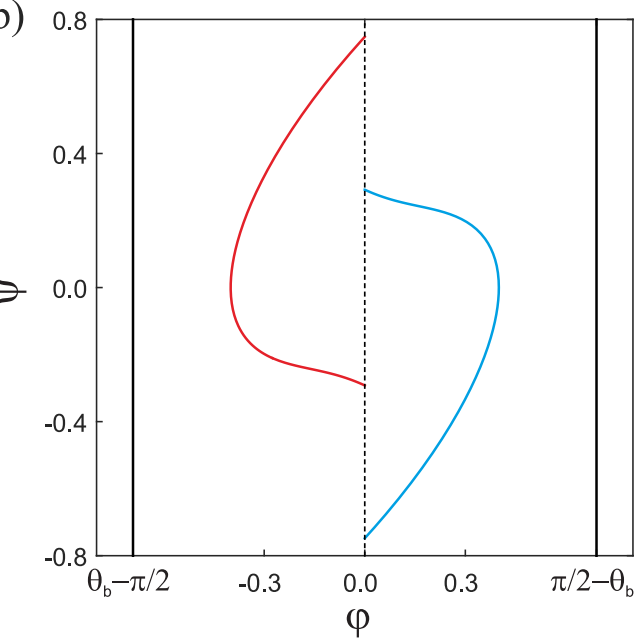

(d)

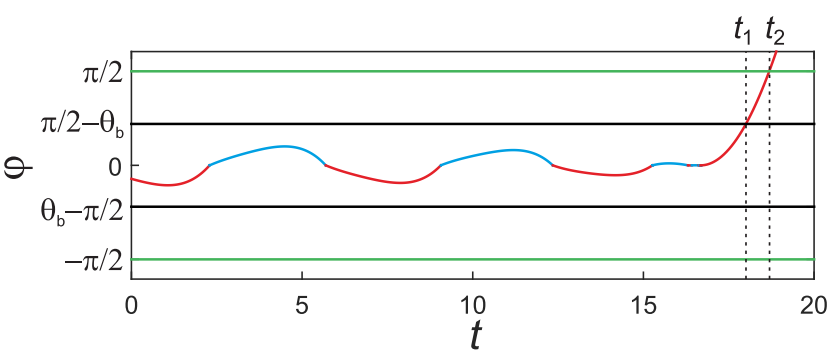

FIG. 3. (a) One-parameter continuation of the periodic response of the rocking block model (9) with respect to the angular frequency $\omega$, computed for the parameter values given in Fig. 2. The solid and dashed branches denote stable and unstable solutions, respectively. The black segment of the bifurcation diagram corresponds to the continuation of the symmetric solution shown in Fig. 2(a), while the green segment results from the continuation of the asymmetric orbit as the one shown here in panel (c). The labels BP $i$ and $\mathrm{SN} i$ stand for branching points and fold bifurcations of limit cycles, respectively. The point GR1 represents a grazing bifurcation of an unstable solution, with the impact boundary $\varphi=0$. The points $\operatorname{SB} i$ represent the parameter values for which the periodic orbit intersects tangentially the static boundaries $\varphi=\theta_{b}-\frac{\pi}{2}$, $\varphi=\frac{\pi}{2}-\theta_{b}$. The orbit shown in panel (b) corresponds to a stable solution computed at the test point P1 $(\omega=0.95)$. The orbit depicted in panel (c) shows a solution for which a tangential contact with the static boundaries occurs, computed at SB2 $(\omega \approx 2.6635)$. The solution depicted in panel (d) corresponds to a block motion where overturning takes place, computed for $\omega=0.902$, a point located to the left of the fold bifurcation SN1 $(\omega \approx 0.9096)$ with initial conditions: $(\varphi, \psi, r, s)=(-0.2254,-0.1597,-0.7778,0.6284)$.

and $\varphi=\frac{\pi}{2}-\theta_{b}$ (see Fig. 1), which can lead to overturning if, for instance, the base excitation suddenly stops. In our numerical investigation, the parameter values given in Table I will be employed, which correspond to a realistic physical scenario.

In Fig. 2 an initial reference solution of the rocking block model (9) is plotted, computed for 
(a)

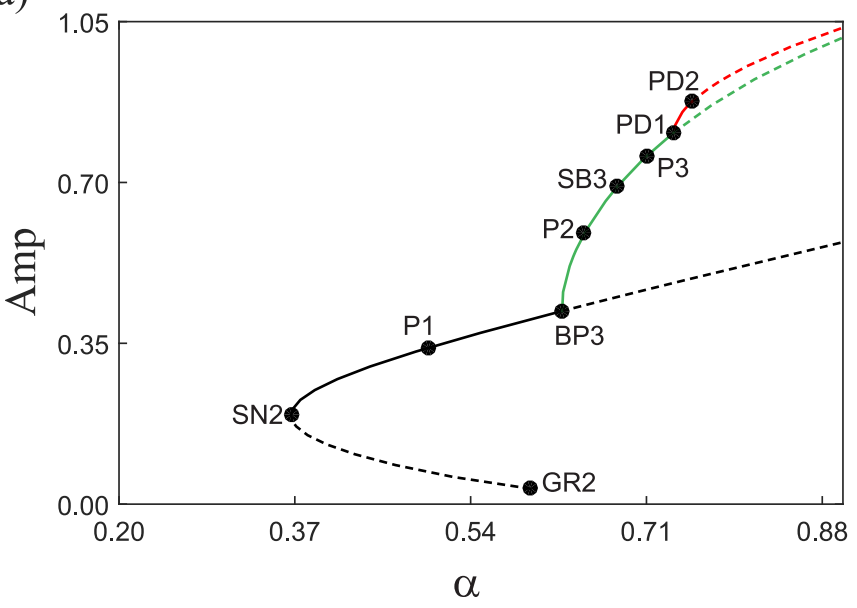

(c)

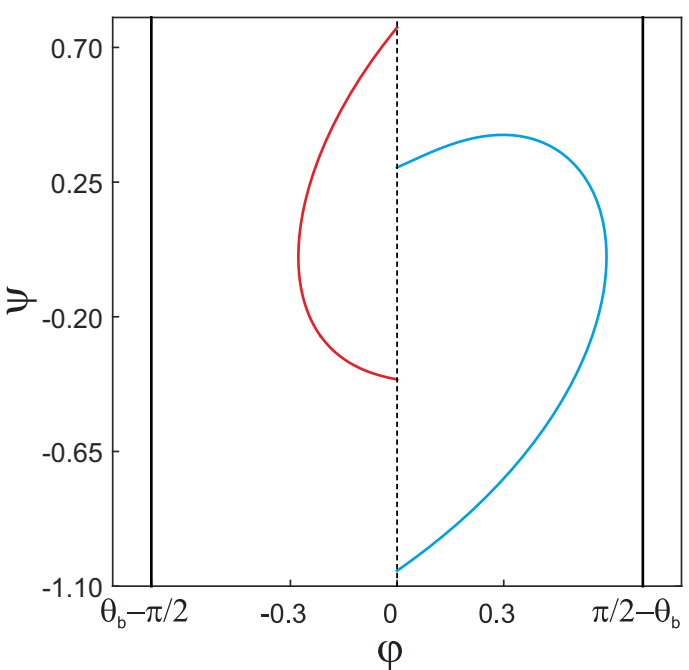

(b)

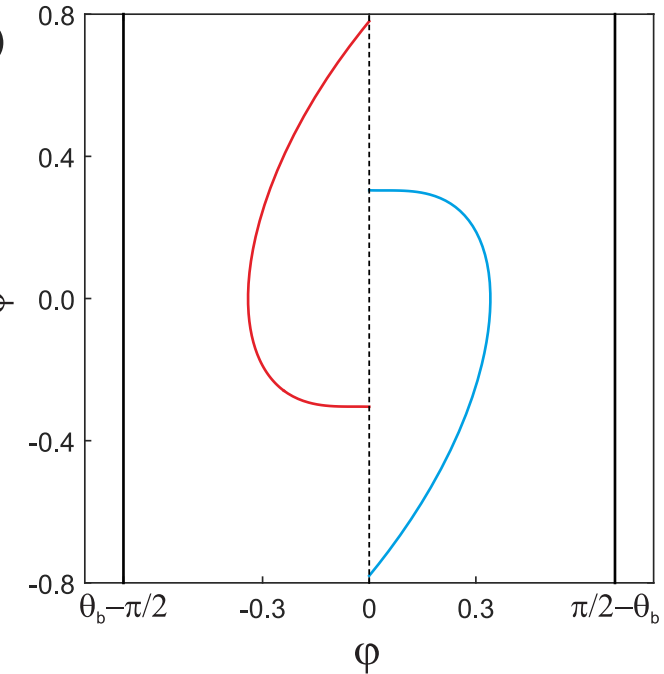

(d)

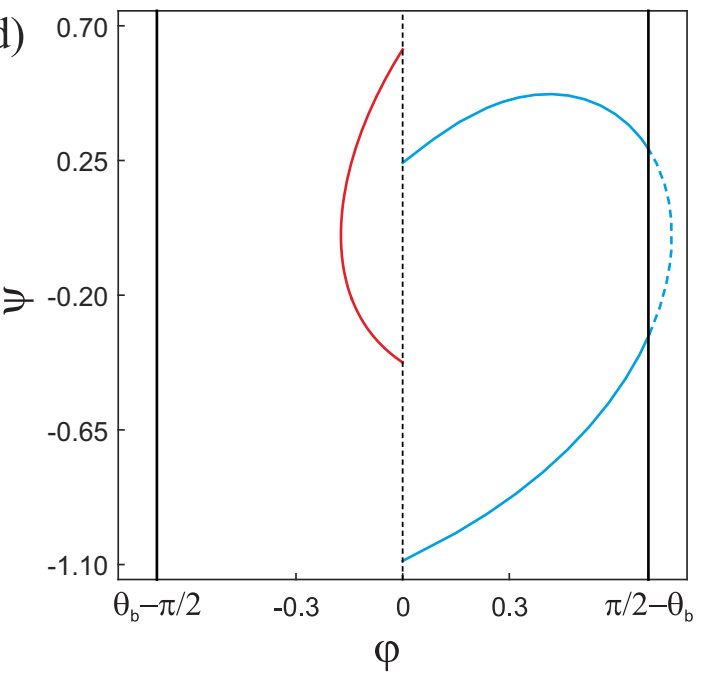

FIG. 4. (a) One-parameter continuation of the periodic response of the rocking block model (9) with respect to the amplitude of excitation $\alpha$, computed for the parameter values given in Fig. 2. The line and color codes are as in Fig. 3. In addition, the red branch represents the numerical continuation of period-2 orbits, which originate from the period-doubling bifurcation PD1 $(\alpha \approx 0.7365)$. Along this branch, a period-doubling bifurcation of a period-2 solution is found at PD2 $(\alpha \approx 0.7542)$. The panels $(b),(c)$ and (d) show phase plots computed at the test points $\mathrm{P} 1(\alpha=0.5), \mathrm{P} 2(\alpha=0.65)$ and $\mathrm{P} 3(\alpha=0.71)$, respectively.

the parameter values given in Table I. This corresponds to a periodic trajectory showing (odd) symmetry, as can be seen from the time plots given in panel (b). As expected, the resulting solution presents points of discontinuity, owing to the restitution law assumed in (3), thereby producing recurrent discontinuities in the angular velocity. At such points (occurring when the rocking block hits the ground $\varphi=0$ ), the angular velocity is instantaneously reduced by a factor $e$, corresponding to the assumed restitution coefficient. This piecewise-smooth periodic solution will be used in our investigation as starting point. 
(a)

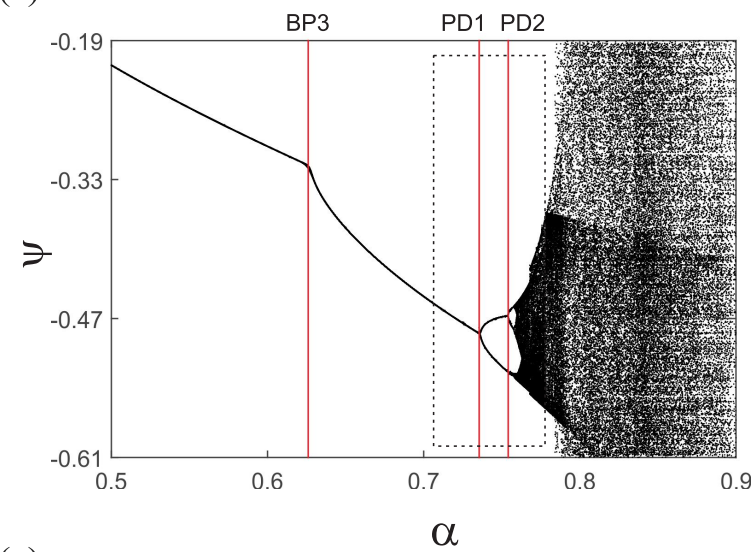

(c)

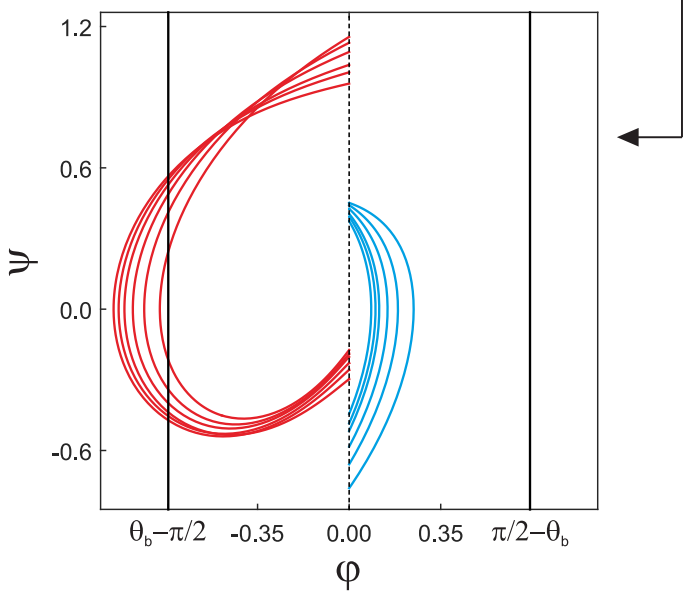

(e)

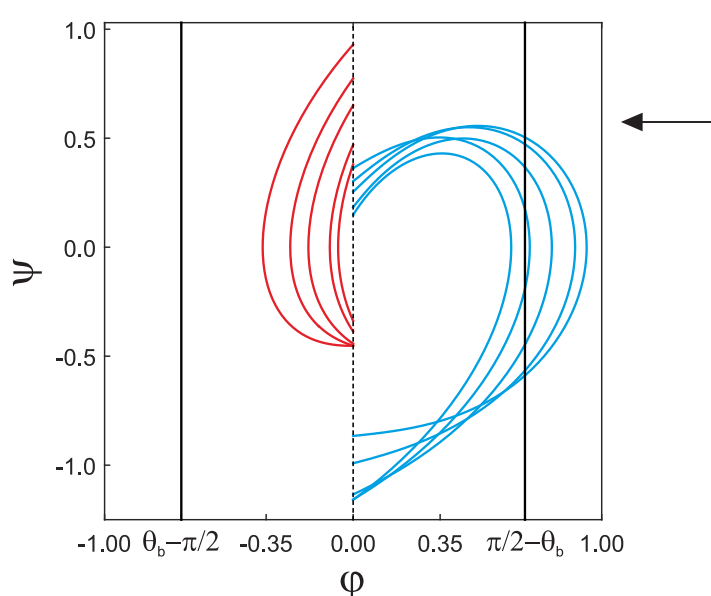

(b)

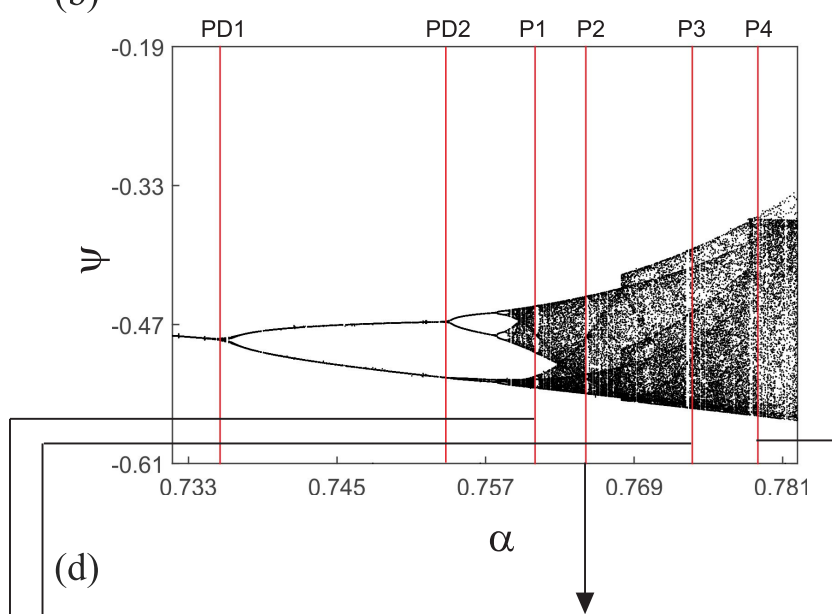

(f)
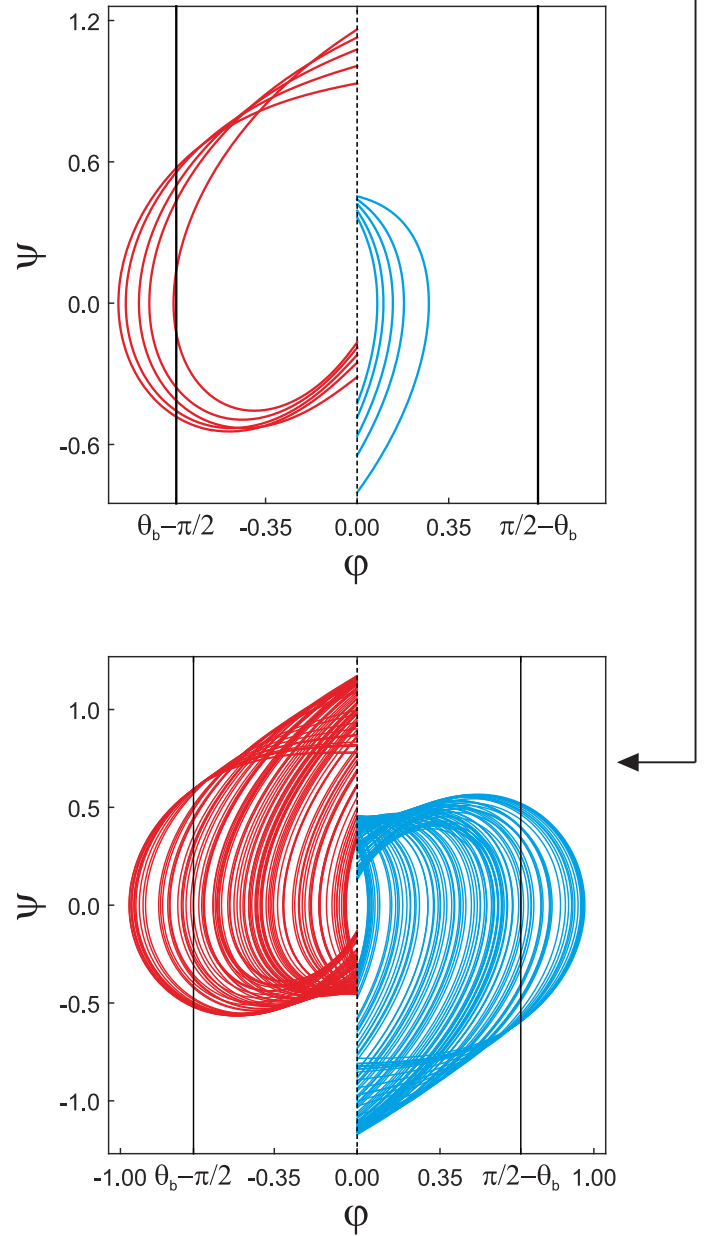

FIG. 5. (a) Bifurcation diagram computed via direct numerical integration of the rocking block model (9), obtained for the same parameter values used in Fig. 4. The vertical red lines mark the bifurcation points BP3, PD1 and PD2 encountered in Fig. 4(a). (b) Blow-up of the boxed region shown in panel (a). Panels (c)-(e) depict different types of periodic solutions found during the computations, obtained for P1 $(\alpha=0.761), \mathrm{P} 2(\alpha=0.7651)$ and $\mathrm{P} 3(\alpha=0.7737)$, respectively. A chaotic solution is plotted in panel (f), for $\mathrm{P} 4(\alpha=0.779)$. 


\section{A. One-parameter analysis of the system response}

As mentioned earlier, the periodic solution depicted in Fig. 2 will be used as starting point for our numerical study. It corresponds to a rocking block motion with odd symmetry, and we will investigate first how this solution is affected when the frequency of ground excitation $\omega$ changes. For this purpose, we will employ numerical continuation methods for non-smooth dynamical systems, implemented via the continuation platform $\mathrm{COCO}^{28}$. The result of this process is presented in Fig. 3, showing the behavior of the solution measure Amp (see (12)) with respect to $\omega$. In panel (a) the resulting bifurcation diagram is depicted, where the black branch represents the continuation of symmetric periodic solutions, as the one shown in panel (b), computed at the test point P1 $(\omega=0.95)$. The solid lines represent branches of asymptotically stable solutions, while the dashed lines stand for families of unstable orbits.

As can be seen in the bifurcation diagram (Fig. 3(a)), the stable branch containing the test point $\mathrm{P} 1$ is limited by the critical points $\mathrm{BP} 1$ and $\mathrm{SN} 1$, corresponding to a branching point and fold bifurcation of limit cycles, respectively. At the branching point, the original stable symmetric solution loses stability, while a solution branch of stable asymmetric periodic orbits is born (green curve), as the one shown in Fig. 3(c). This green branch persists for a wide window of $\omega$-values and terminates at another branching point BP2. Here, the asymmetric solutions disappear and the family of symmetric solutions (black branch) recovers stability. In addition, two critical points SB1 and SB2 are detected during the numerical continuation, corresponding to $\omega$-values for which the periodic solution makes tangential contact with the critical overturning boundary $\varphi= \pm\left(\theta_{b}-\frac{\pi}{2}\right)$. In this way, we can identify a branch in the bifurcation diagram (between SB1 and SB2) where overturning can occur, as discussed before. An example of such a critical solution is given in Fig. $3(\mathrm{c})$, computed at SB2 $(\omega \approx 2.6635)$.

Next, we will analyze the behavior of the rocking block when the amplitude of the base excitation $\alpha$ is varied, while keeping the excitation frequency constant. As before, we will carry out the investigation via numerical continuation methods for non-smooth dynamical systems, using the solution shown in Fig. 2 as starting point. The result of this process is displayed in Fig. 4. In this case, we identify three different branches, plotted in black, green and red. The black and green curves, as before (see Fig. 3), represent families of symmetric and asymmetric periodic solutions, respectively. These two curves intersect each other at a branching point BP3 found for $\alpha \approx 0.6279$. On the green branch (asymmetric solutions) two additional critical points are found, 
labeled SB3 ( $\alpha \approx 0.6819$ ) and PD1 ( $\alpha \approx 0.7365)$, where the periodic solution touches tangentially the overturning boundary $\varphi= \pm\left(\theta_{b}-\frac{\pi}{2}\right)$ and a period-doubling bifurcation of limit cycles occurs, respectively. At the PD1 point the original period-1 solution loses stability, while a branch of stable period-2 orbits is born. The numerical continuation of these orbits is given by the red branch shown in Fig. 4. Further numerical investigations reveal that the period-2 solution bifurcates once more via another period-doubling point ( $\mathrm{PD} 2, \alpha \approx 0.7542$ ), hence producing periodic solutions with four times the original period.

In order to gain a deeper understanding of the dynamics of the rocking block model (9), we will carry out a parametric investigation of the system via direct numerical integration as follows. First, we fix an initial value for the amplitude $(\alpha=0.5)$ and then integrate the system over 300 periods of excitation in order to eliminate transients. Then, we extend the numerical integration for a range of 100 periods and store samples of the obtained solution at every $t=0,2 \pi, 4 \pi, \ldots$, after which $\alpha$ is increased by a small amount and the process is repeated, using the last sample as starting value for the next step. The result of this numerical process is presented in Fig. 5(a) and (b), showing the angular velocity $\psi$ on the vertical axis. The picture confirms the numerical predictions obtained via numerical continuation described above, in particular the period-doubling bifurcations detected during continuation (labeled PD1 and PD2). As can be seen in Fig. 5(b), the numerical investigation reveals the presence of a period-doubling cascade producing periodic solutions of increasing period, leading to chaotic behavior, as shown in Fig. 5(c)-(f).

\section{B. Two-parameter analysis of the system response}

In the previous section, our main concern was to investigate the behavior of the rocking block model (9) under one-parameter perturbations, in particular with respect to the frequency $\omega$ and amplitude $\alpha$ of the ground oscillation. This study revealed the presence of critical parameter values (corresponding for instance to period-doubling and fold bifurcations) upon which the system dynamics suffers significant changes. In the present section our main goal will be to investigate how these critical points are distributed in the $\alpha-\omega$ plane, so as to be able to classify the system dynamics with respect to the excitation parameters. For this purpose, we will employ COCO's numerical routines for the two-parameter continuation of codimension-1 bifurcations. Specifically, we will carry out the two-parameter continuation of the critical points found in Figs. 3(a) and 4(a), with respect to $\omega$ and $\alpha$. 
(a)

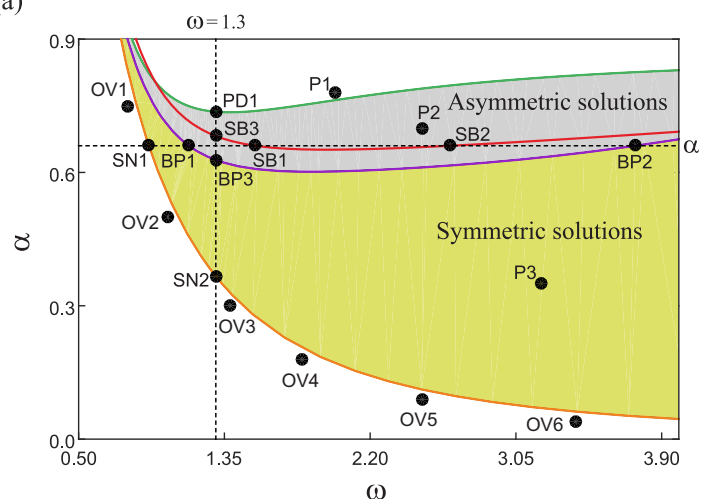

(c)

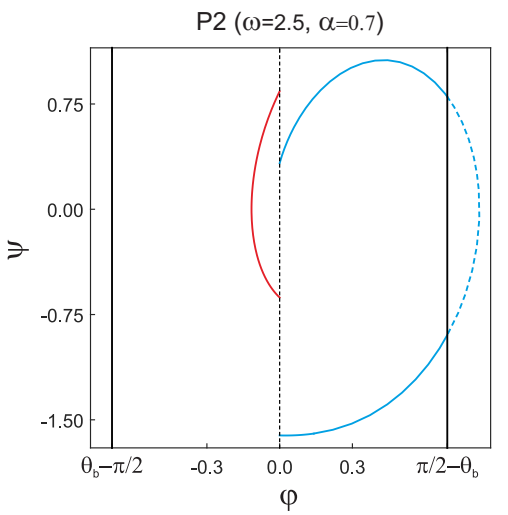

(e)

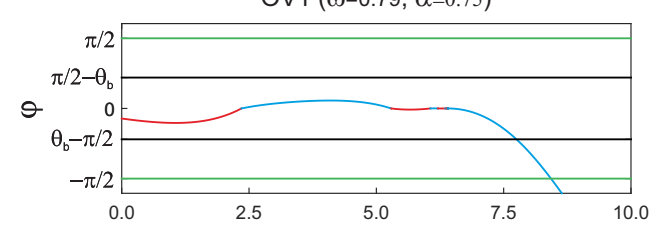

OV2 $(\omega=1.02, \alpha=0.5)$

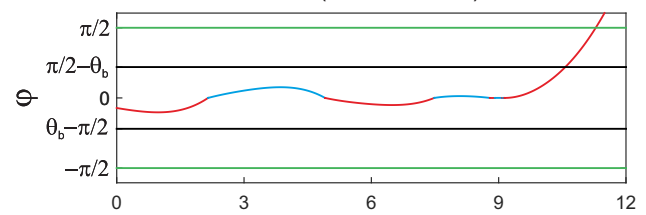

OV3 $(\omega=1.38, \alpha=0.3)$

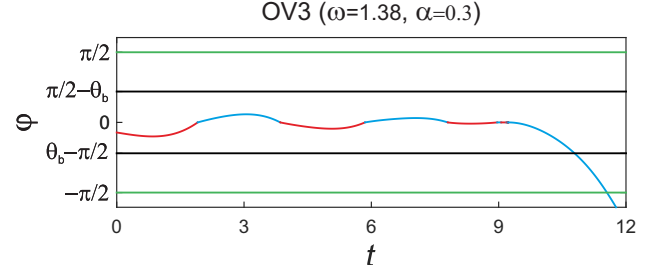

(b)

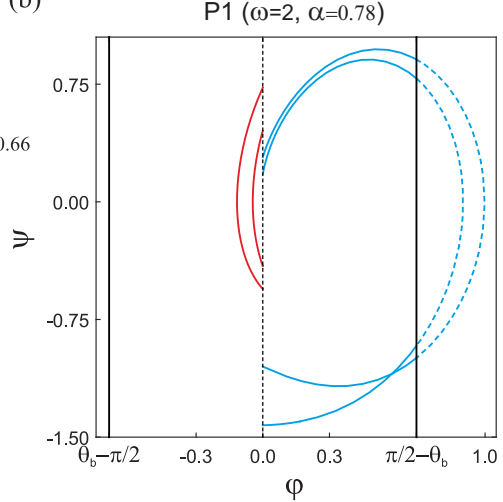

(d)

P3 $(\omega=3.2, \alpha=0.35)$

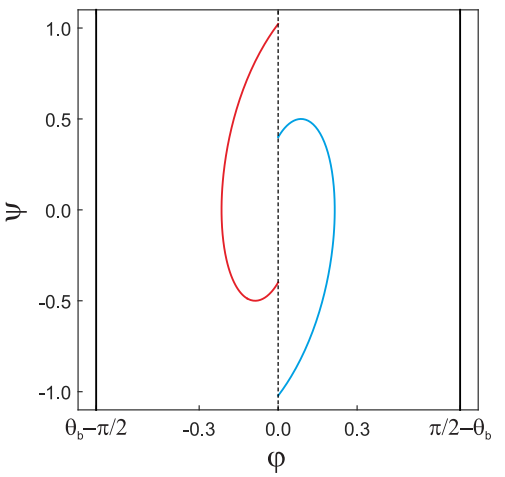

OV4 $(\omega=1.8, \alpha=0.18)$
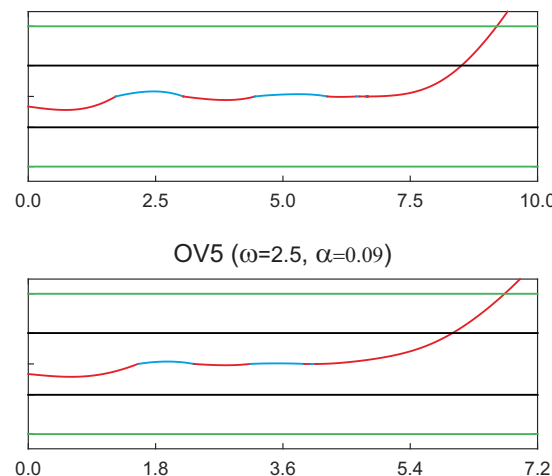

OV6 $(\omega=3.4, \alpha=0.04)$

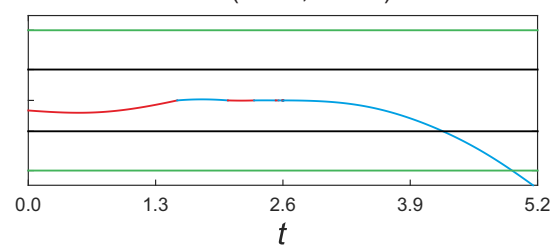

FIG. 6. (a) Two-parameter continuation of the PD (green), SB (red), BP (purple) and SN (orange) points, found in Figs. 3(a) and 4(a), with respect to $\omega$ and $\alpha$. The intersections of the horizontal $(\alpha=0.66)$ and vertical $(\omega=1.3)$ lines with the depicted curves correspond to some of the critical points shown in Figs. 3(a) and 4(a), respectively. The grey area represents the parameter region in which stable (period-1) asymmetric solutions exist, as shown in panel (c). The yellow area gives the parameter values yielding stable (period-1) symmetric solutions, as depicted in panel (d). Panel (b) shows a period-2 solution originating from the period-doubling bifurcation found along the green curve. The time plots presented in panel (e) correspond to solutions crossing the critical boundary $\varphi= \pm \frac{\pi}{2}$ where overturning occurs (initial conditions for all time plots: $(\varphi, \psi, r, s)=(-0.2254,-0.1597,-0.7778,0.6284))$. 
The outcome of the numerical procedure described above can be found in Fig. 6. Panel (a) presents the resulting bifurcation diagram showing the continuation of period-doubling (green curve), branching points (purple curve) and fold (orange curve) bifurcations. In addition, a red curve is included that corresponds to the two-parameter continuation of the points $\mathrm{SB} i$ found before, which gives a parameter-dependent family of period-1 solutions making tangential contact with the overturning boundary $\varphi= \pm\left(\theta_{b}-\frac{\pi}{2}\right)$. Consequently, this red curve provides critical information regarding the rocking block dynamics, as in this way we can determine combinations of frequency and amplitude of the ground oscillation that may lead to overturning of the structure. Panels (c) and (d) in Fig. 6 illustrate the meaning of this curve, giving examples of period-1 solutions with and without overturning possibility, respectively. These solutions also demonstrate the meaning of the purple curve shown in the bifurcation diagram, which corresponds to the twoparameter continuation of the branching points (labeled BP) found in Figs. 3(a) and 4(a). As explained before, this bifurcation defines the connection between symmetric and asymmetric period1 solutions, where the region producing such types of solutions is colored in yellow and grey in Fig. 6(a), respectively. In panel (b), a phase portrait of a period-2 solution is depicted, owing its presence to the period-doubling phenomena encountered in the system (green curve). Moreover, notice that the numerical continuation of period-doubling (green curve) and fold (orange curve) bifurcations define a region in the $\omega-\alpha$ space (see Fig. 6(a)) of stable period-1 responses without any crossing with the critical boundary $\varphi= \pm \frac{\pi}{2}$, where overturning takes place. Further numerical tests (shown in Fig. 6(e)) reveal that below the fold curve the system presents aperiodic responses where overturning occurs, as confirmed at the test points OV1-OV6 shown in the figure. In this way, the computed fold curve can also serve as a critical boundary in the parameter space so as to determine under which oscillation conditions overturning phenomena can be avoided.

\section{BASIN STABILITY ANALYSIS}

In this section, we analyze previously presented results using basin stability approach. As aforementioned, in case of single degree-of-freedom system, considered in this paper, the basin stability approach can be used to calculate basins of attractions when we draw two initial conditions (for fix parameter values of the system). Before the analysis of obtained results, we list solutions types that exist in the system:

- Equilibrium - rocking block is not moving and $\varphi=\psi=0.0$, 
(a)

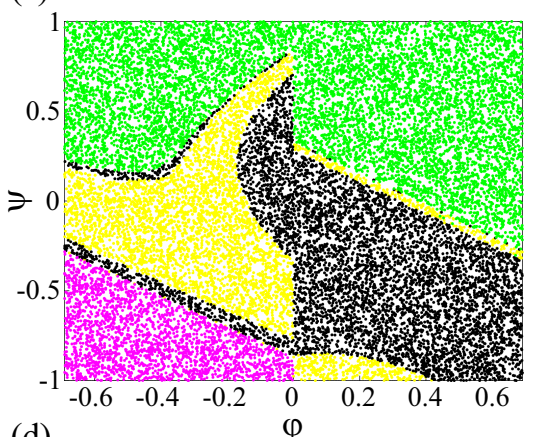

(d)

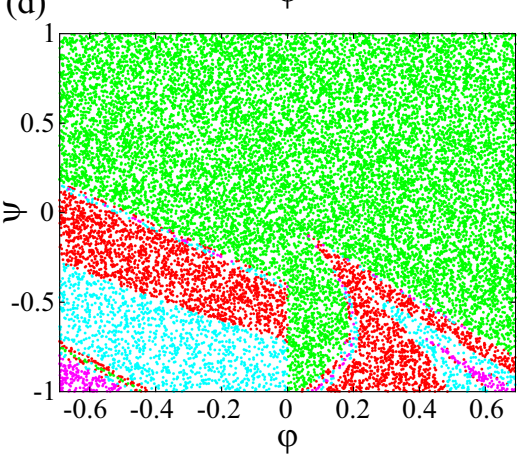

(b)

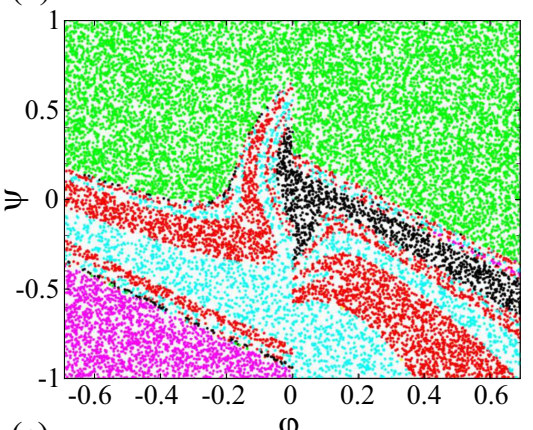

(e)

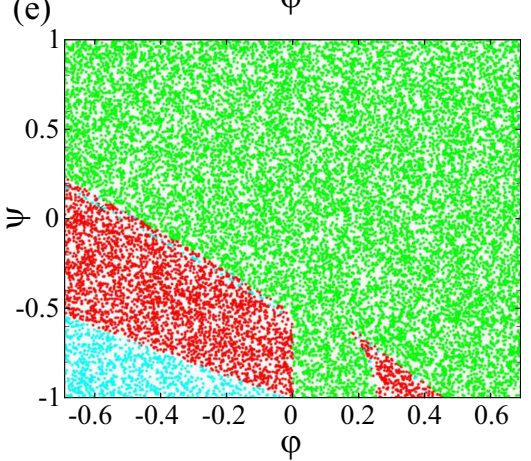

(c)

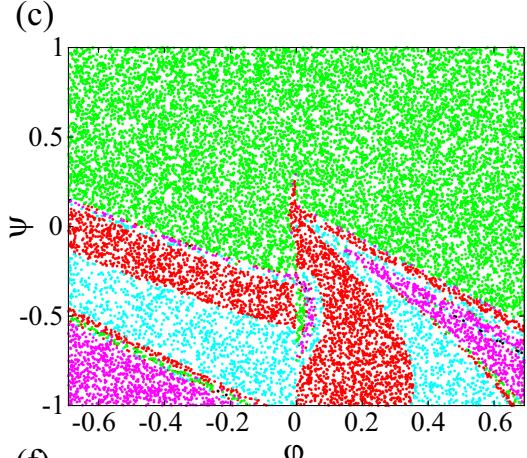

(f)

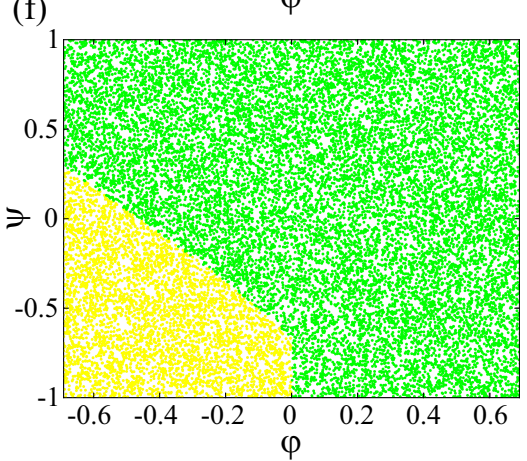

- Period 1 asym + Period 1 - Overturn $+\pi / 2 \cdot$ Chaos and period $>2$

- Period 1 asym - $\quad \times$ Period $2 \cdot$ Overturn $-\pi / 2 \bullet$ Equlibrium

FIG. 7. Basins of attractions calculated using basin stability method for alpha $=0.66$ and varying $\omega$ equal to 1.0 (a), 1.16 (b), 1.5 (c), 2.0 (d), 3.0 (e), 4.0 (f). Initial parameters are drawn from $\varphi \in\left(-\pi / 2+\theta_{b}, \pi / 2+\right.$ $\left.\theta_{b}\right)$ and $\psi \in(-1,1)$. Colors of dots determine solutions.

- Period 1 symmetric solution - rocking block is oscillating periodically with symmetric orbit,

- Period 1 asymmetric solution - period 1 solution is shifted in a positive $\phi$ direction,

- Period 1 asymmetric solution - period 1 solution is shifted in a negative $\phi$ direction,

- Period 2 solution bifurcating from both asymmetric solutions (we do not distinguish the asymmetric solution that it bifurcates),

- Chaotic solutions or periodic with a period above 2,

- Overturning solution where the block reaches $\phi=\pi / 2$,

- Overturning solution where the block reaches $\phi=-\pi / 2$,

Hence, we distinguish eight solutions. Due to the very narrow range of existence of solutions with a higher period then 2 , we decide to present them together with chaotic oscillations. 
To have overview of the structure of the phase space we perform analysis for six selected values of $\omega(\omega=1.0, \omega=1.16, \omega=1.5, \omega=2.0, \omega=3.0$ and $\omega=4.0)$ and constant value of the amplitude $\alpha=0.66$ (see bifurcation diagram in Fig. 4 and horizontal line in Fig. 6). In Fig. 7 we show calculated basins of attractions. Initial conditions are drawn from ranges: $\varphi \in(-\pi / 2+$ $\left.\theta_{b}, \pi / 2+\theta_{b}\right)$ and $\psi \in(-1,1)$ and for each diagram we calculate 20000 Bernoulli trials. For $\omega=1.0$ (panel (a)) we observe period 1 symmetric solution shown in yellow color. Most likely, it occurs when the initial position is negative. For larger values of the initial velocity, we observe domination of the overturning solution $(\varphi=\pi / 2$, green area), while for negative initial position and velocity we see the overturning of the block in the opposite direction $(\varphi=-\pi / 2)$ indicated by purple area. Moreover, we observe a large range where the block is stable in the equilibrium position and does not oscillate. In panel (b) the value of the frequency of excitation is equal to $\omega=1.16$, and it is above the BP bifurcation line. Hence, we observe two period 1 asymmetric solutions (red and blue colors). Similarly, as for $\omega=1.0$, positive values of the initial velocity results in the overturning of the block. The range where the block reaches the equilibrium position shrinks significantly, and this solution is less probable. Next, the plot (c) is computed for $\omega=1.5$, and we see that with the increase of the excitation frequency, the probability of the overturning is higher, while the equilibrium solution is no longer observed. For negative values of the initial velocity, we see the domination of the period 1 asymmetric solution. Next, for $\omega=2.0$ (panel (d)) we see that the range of the overturning in the positive direction $(\varphi=\pi / 2)$ is still increasing, while the overturning via negative $\varphi$ has a very low probability of occurrence. Other solutions are moved toward negative values of the initial velocity. The same tendency is observed with a further increase of $\omega$ presented in panels (e,f). The result presented in the last panel (f) is calculated for $\omega=4.0$, which is below the BP bifurcation line; hence we observe period 1 symmetric solution (yellow area). Summarizing, the basins of attraction are compact and only at their borders, we observe complex structures. We calculate the probability of reaching all considered solutions. For values of $\omega$ for which we observe asymmetric solutions, probabilities of their occurrence are equal, hence neither of them is more likely to be observed assuming random initial state. For the higher frequency of excitation, the probability of overturning the block increases and this solution becomes dominant. For the analyzed set of the system parameters, many solutions coexist; hence the system is multistable. Based on convergence analysis, we see that it is enough to calculate 5000 Bernoulli trials to have precise information about the phase space structure (we show more to have a densly filled plot). Please note that important factor which influence the final solution is the form 
of the excitation function. In this paper, the excitation amplitude is harmonically modulated by $\sin (\omega \widetilde{t})$ function, which in the first phase of motion moves block into the right direction, hence when the initial velocity is positive it can more easily cause the block to overturn.
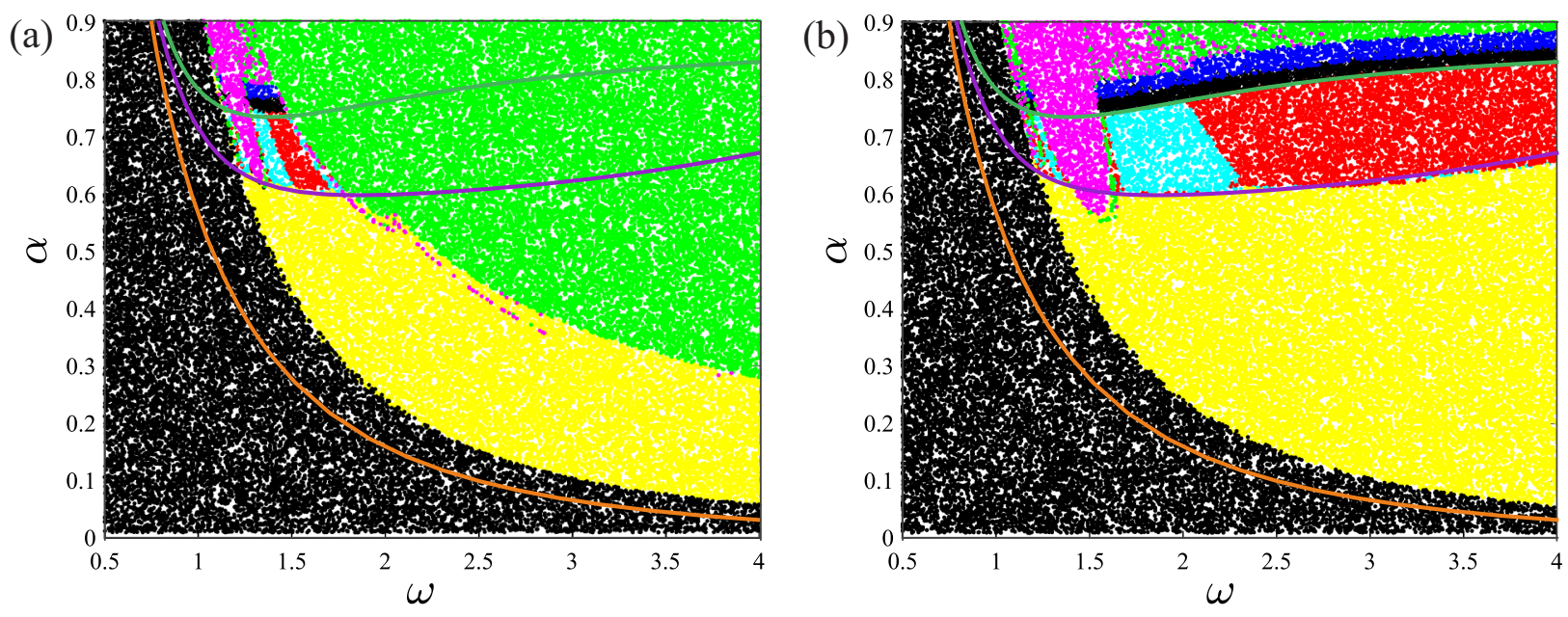

- Period 1 asym $+\quad$ Period 1 - Overturn $+\pi / 2 \bullet$ Chaos and period $>2$

- Period 1 asym - $\quad \times$ Period 2 - Overturn $-\pi / 2 \bullet$ Equlibrium

FIG. 8. Two dimensional diagrams for varying $\alpha$ and $\beta$ with constant initial conditions $(\varphi=0.01, \psi=0.0)$ and two values of phase of the exaction $\beta=0$ (a) and $\beta=\pi / 2$ (b). Solid lines correspond to SN (orange), $\mathrm{BP}$ (purple) and PD (green) bifurcations, respectively.

In next step we analyze the response of the system in two-parameter space $(\alpha, \omega)$ in the following ranges: $\alpha \in(0.1,0.9)$ and $\omega \in(0.5,4.0)$ for fixed initial conditions: $\varphi=0.01$ and $\psi=0.0$. Hence, the block is slightly angled into the positive $\varphi$ side. It allows to study which solutions are reachable from values of initial conditions close to the equilibrium position, which has practical importance. In the description of the previous figure, we conclude that the initial phase of the excitation significantly influences the response of the system; thus we also take it into account in our calculations. We introduce an initial phase of excitation $\beta$ to harmonic modulation of the excitation function: $\sin (\omega \widetilde{t}+\beta)$ (see Eqs (6) and (7)). The diagram obtained for $\beta=0$ is shown in Fig. 8 (a) and for $\beta=\pi / 2$ in Fig. 8(b). To compare the ranges of solutions' stability calculated based on the basin stability method and the path-following analysis, we copy bifurcations lines from Fig. 6 - namely the SN (orange), the BP (purple) and the PD (green) line. In both plots, we do not see the difference in the range where the equilibrium solution (black dots) exists. However, based on bifurcation lines, we see the significant changes above the SN line. The basins od attractions of other solutions for the two considered values of $\beta$ differ significantly. In panel (a) above the equilibrium solution, we observe the range of period 1 symmetric solution (yellow dots) which is 

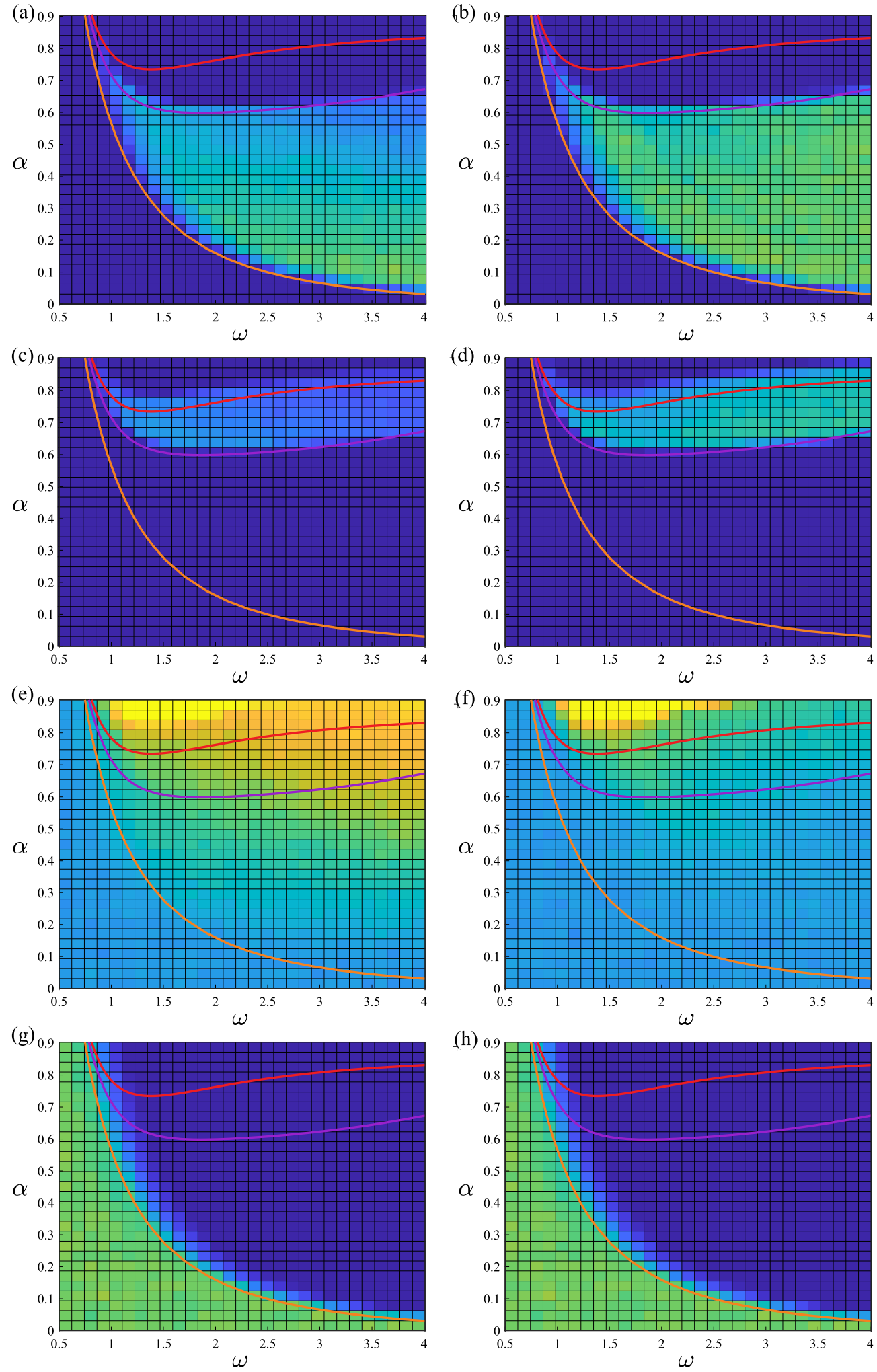

Probability

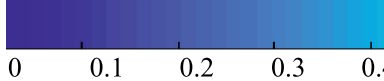

FIG. 9. Probability of occurrence of four solutions: period 1 symmetric (a,b), period 1 asymmetric (c,d), two overturning (e,f) and equilibrium (g,h) for the phase of the excitation $\beta=0$ (left column) and $\beta=\pi / 2$ (right column) in $\alpha$ and $\omega$ parameters space. Solid lines correspond to SN (orange), BP (purple) and PD (green) bifurcations, respectively. 
bounded from the top by the BP bifurcation line and the overturning solution (green dots). Above the BP bifurcation line we should observe the period 1 asymmetric solutions (red and blue dots), but they are present only in a very narrow range. This confirms that the selected phase of excitation leads to overturning of the block, especially for higher values of the excitation frequency. Above the PD bifurcation line, we see the appearance of period 2 asymmetric solutions (we do not distinguish the) and then fast transition to chaos via period doubling sequence. Above the chaotic range, we observe overturning. For the second value of the excitation phase $\beta=\pi / 2$ (panel (b)) period 1 symmetric solution is observed nearly in the whole range above the equilibrium solution and below the BP bifurcation line (only in narrow range the overturning solution appears - purple dots). Between the BP and the PD lines, period 1 asymmetric solutions (red and blue dots) exist above $\omega \approx 1.7$, while below this value, we observe block overturning (purple dots). Crossing of the PD bifurcation line results in a transition to period 2 solution and then via period doubling sequence appearance of a chaotic solution. Further increase of $\alpha$ leads to overturning of the block (green and purple dots). Presented plots show that the initial phase of the excitation is crucial for the stability of the block. Hence, the initial phase of motion strongly affects the shape of basins of attractions and the final state of the rocking block. The exception is the equilibrium solution that is not sensitive to the initial phase of the excitation $\beta$.

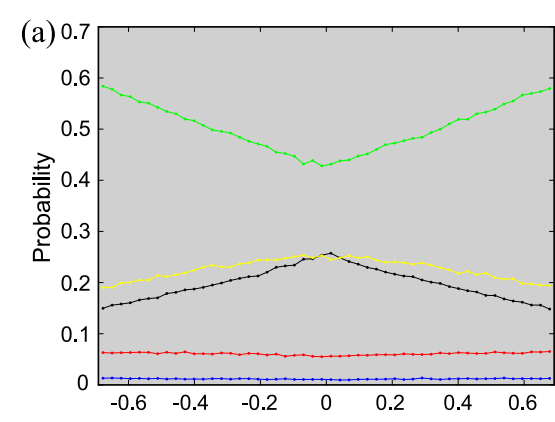

$\varphi$

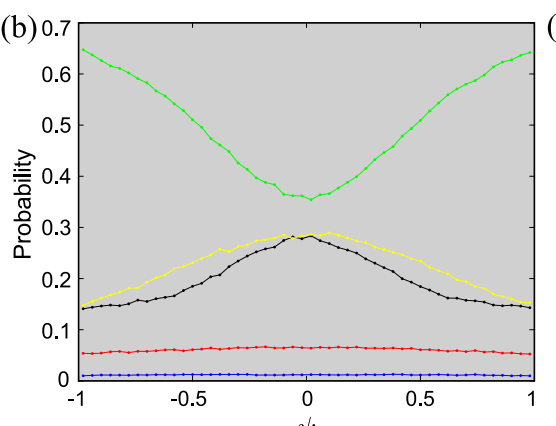

Overturn - Equilibrium

Period 1 symmetric —Period 1 asymmetric —Other solutions

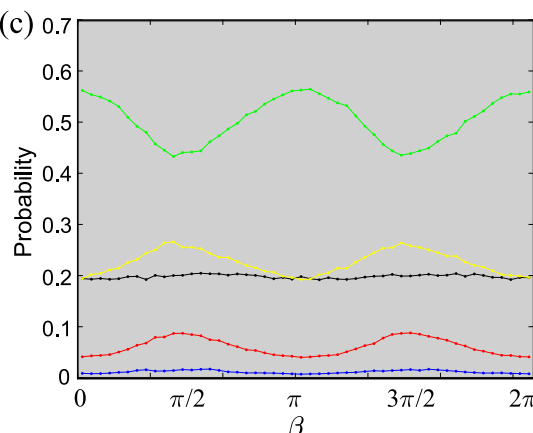

FIG. 10. Probability of occurrence of five solutions for random initial conditions $\left(\varphi \in\left(-\pi / 2+\theta_{b}, \pi / 2+\right.\right.$ $\left.\theta_{b}\right)$ and $\psi \in(-1,1)$ ) and parameters $(\alpha \in(0.1,0.9), \omega \in(0.5,4.0)$ and $\beta \in(0.0,2 \pi))$ as a function of the initial deflection (a), the initial velocity (b) and the phase of the excitation $\beta$ (c).

The subsequent analysis is devoted to the study of probability to reach previously presented solutions. The obtained results are shown in Fig. 9. We draw initial conditions $(\varphi \in(-\pi / 2+$ $\left.\theta_{b}, \pi / 2+\theta_{b}\right)$ and $\left.\psi \in(-1,1)\right)$ and two parameters values $(\alpha \in(0.1,0.9), \omega \in(0.5,4.0))$ for two values of the phase of the excitation ( $\beta=0.0$ (right column) and $\beta=\pi / 2$ (left column)). For 
each value of $\beta$, we perform 1,000,000 Bernoulli trials, and we show probability plots in $(\omega-\alpha)$ plane for four solutions, namely, the period 1 symmetric, the period 1 asymmetric (two types together), the overturning (without distinguishing direction) and the equilibrium. We do not show periodic solutions with period higher than 2 as well as neither periodic solutions because their probabilities are low in comparison to the presented ones. To show the probability of occurrence of each solution, we divide the whole range into 900 cells (30x30), and we calculate the probability to reach given solution for each cell. We also add the SN, the BP and the PD bifurcations lines obtained via path-following. In panels $(a, b)$ we present the period 1 symmetric solution, for which the phase of the excitation $\beta$ has a significant influence. For $\beta=0$ it is less probable then for $\beta=\pi / 2$. Moreover, for $\beta=0$ it exists closer to the SN bifurcation line then for $\beta=\pi / 2$, which is equally probable in the whole existence range. Next, we consider the period 1 asymmetric solutions (panels $(\mathrm{c}, \mathrm{d})$ ) and we see that for $\beta=\pi / 2$ they are more likely to occur and the probability is equally spaced between the bifurcations lines. Then, we investigate the occurrence of the overturning solution (panels (e,f)). We see that for $\beta=0$ with an increase of $\alpha$, the overturning solution starts to dominate, and at the top of the plot, it is the only existing solution. In case of $\beta=\pi / 2$ the overturning is less probable and its higher probability is reached for $\alpha>0.8$ and between $\omega=1$ and $\omega=3$. Last two panels (g,h) show the equilibrium solution. We see that there is no visible difference between both plots; hence, we claim that stabilization of the block in the steady-state is independent on the phase of the excitation $\beta$. The main conclusion from this analysis is the importance of initial phase of excitation that has a dominating role and significantly changes the response of the system.

In the last considered case, we assume random initial phase of excitation. The results are shown in Fig. 10. We draw initial conditions $\left(\varphi \in\left(-\pi / 2+\theta_{b}, \pi / 2+\theta_{b}\right)\right.$ and $\left.\psi \in(-1,1)\right)$ and three parameters $(\alpha \in(0.1,0.9), \omega \in(0.5,4.0)$ and $\beta \in(0.0,2 \pi))$ and we perform $2,000,000$ Bernoulli trials. We show the probability of five solutions (overturning, equilibrium, period 1 symmetric, period 1 asymmetric and all other responses) as a function of the initial deflection (panel (a)), the initial velocity (panel (b)) and the phase of the excitation (panel (c)). For that purpose, we divide the span of a given parameter in 50 equal ranges and in each range we calculate probabilities to reach all considered solutions. The number of trials in each section is approximately the same (40,000 with standard deviation 242). For both initial conditions, we observe an increase in the probability of the overturning solution when their values move away from zero. The opposite tendency is observed for period 1 symmetric and equilibrium solutions. The last two solutions 
are nearly not affected by initial conditions imposed on the rigid block. In panel (c), we see the significant dependence of solutions' probabilities on parameter $\beta$. It is visible that when the excitation starts from zero and increase in positive $(\beta \approx 0)$ or in negative $(\beta \approx \pi)$ the overturning occurs more likely than in case when the excitation starts from maximum $(\beta \approx \pi / 2)$ or minimum ( $\beta \approx 3 \pi / 2$ ) values. A different response is observed for the period 1 symmetric and asymmetric solutions. Equilibrium and other solutions are not affected by parameter $\beta$.

\section{CONCLUDING REMARKS}

This paper carried out a detailed numerical study of the dynamical response of a rocking block under ground motion. The considered physical model can represent several practical scenarios, such as buildings, silos, containers, scaffoldings, and free standing power transformers. One of the main concerns of this work was to determine the conditions under which overturning of the underlying structure may occur. Therefore, special attention was paid to the periodic solutions of the system and their evolution under the variation of base excitation. To study the behavior of the model, two different types of numerical approaches, namely direct numerical integration and pathfollowing techniques for non-smooth dynamical systems, were adopted. The path-following analysis via COCO revealed the presence of critical parameter values (corresponding for instance to period-doubling and fold bifurcations) upon which the system dynamics suffer significant changes. In addition, branching points leading to symmetry breaking were detected, where families of symmetric and asymmetric periodic solutions collide. During this study, two critical boundaries were defined $\left(\varphi= \pm\left(\theta_{b}-\frac{\pi}{2}\right)\right.$, labeled $\left.\mathrm{SB} i\right)$, which give the critical angles beyond which overturning can occur, for instance, when the base excitation suddenly stops. In this way, it is possible to identify ground oscillation regimes with a risk of overturning.

Our work significantly extended results presented by Hogan ${ }^{7,9,14}$. The majority of his investigations were performed for the slander structures. Such assumption allows to linearize equations of the motion and applies analytical methods. While, in our work, we assume that the ratio between the breadth and height of the structure is equal to 1.21 , which is a typical value for this class of systems. Based on comparing our work with Hogan's results, we see that the structure's slenderness significantly influences the system's response, thus showing the dynamics of the real structure one has to study the full nonlinear model in this paper. Due to the limitation of the numerical methods adopted in Hogan's works, he showed a few simulations of non-slander systems' behavior. Hence, 
he didn't present a complete view of the dynamics in the frequency and amplitude of the excitation space. Our numerical investigation revealed the complex dynamical behavior of the rocking block model, in particular due to the presence of period-doubling cascades producing periodic solutions of increasing period, leading to chaotic motion. Further studies were carried out using two-parameter continuation of the codimension-1 bifurcations found before, using the frequency and amplitude of base excitation as the main control parameters. A critical curve corresponding to the two-parameter continuation of the points $\mathrm{SB} i$ found in one-parameter analysis was identified. It gave a parameter-dependent family of period-1 solutions making tangential contact with the overturning boundary $\varphi= \pm\left(\theta_{b}-\frac{\pi}{2}\right)$. This curve provides critical information regarding the dynamics of the rocking block, since in this way a combination of frequency and amplitude of the ground oscillation which may lead to overturning of the structure can be determined. The results from the path-following method are supported with the basin stability analysis. We studied the coexistence of solutions and the influence of the system parameters and initial conditions on the probability of their occurrence. These are the further developments of this work based on Hogan's study $^{7,14}$, where he reported existence of such behavior in the rocking block. We found that in the considered range of the amplitude and the frequency of the excitation, the system is mostly multi-stable and final solution strongly depends on initial conditions and initial phase of excitation. It is especially essential from practical point of view because we usually do not have control on the excitation signal. Thus, the parameters of the block should be carefully chosen based on most probable excitation function.

This study provides crucial information regarding the periodic and non-periodic response of the rocking rigid block subjected to excitation function with varying parameters. The obtained results can be used for development and practical applications to obtain given type of stable oscillations or prevent overturning under sea waves excitation.

\section{ACKNOWLEDGMENTS}

This work has been supported by National Science Centre, Poland - Project No. 2018/31/D/ST8/02439 (P. Brzeski and P. Perlikowski).

Compliance with ethical standards. Conflict of interest. The authors declare that they have no conflict of interest concerning the publication of this manuscript. Data accessibility. The data that support the findings of this study are available from the corresponding author upon reasonable 
request.

\section{REFERENCES}

${ }^{1}$ C. Yilmaz, M. Gharib, and Y. Hurmuzlu, "Solving frictionless rocking block problem with multiple impacts," Proc. R. Soc. A 465, 3323-3339 (2009).

${ }^{2}$ E. Avgenakis and I. Psycharis, "Complex resonse of a rocking block to a full-cycle pulse," Journal of Engineering Mechanics 146, 04020020 (2020).

${ }^{3}$ Y. Bao and D. Konstantinidis, "Dynamics of a sliding-rocking block considering impact with an adjacent wall," Earthquake Engineering and Structural Dynamics 49, 498-523 (2020).

${ }^{4} \mathrm{G}$. Housner, "The behavior of inverted pendulum structures during earthquakes," Bulletin of the Seismological Society of America 53, 403-417 (1963).

${ }^{5}$ C. Yim, A. Chopra, and J. Penzien, "Rocking response of rigid blocks to earthquakes," Earthquake Engineering and Structural Dynamics 8, 565-587 (1980).

${ }^{6}$ Y. Ishiyama, "Motions of rigid bodies and criteria for overturning by earthquake excitations," Earthquake Engineering and Structural Dynamics 10, 635-650 (1982).

${ }^{7}$ S. Hogan, "On the dynamics of rigid-block motion under harmonic forcing," Proc. R. Soc. A 425, 441-476 (1989).

${ }^{8}$ S. Yim and H. Lin, "Chaotic behavior and stability of free-standing offshore equipment," Ocean Engineering 18, 225-250 (1991).

${ }^{9}$ S. Hogan, "The effect of damping on rigid block motion under harmonic forcing," Proc. R. Soc. A 437, 97-108 (1992).

${ }^{10}$ F. Pena, F. Prieto, P. Lourenco, A. Campos Costa, and J. Lemos, "On the dynamics of rocking motion of single rigid-block structures," Earthquake Engineering and Structural Dynamics 36, 2383-2399 (2007).

${ }^{11}$ E. Dimitrakopoulos and M. DeJong, "Revisiting the rocking block: closed-form solutions and similarity laws,” Proc. R. Soc. A 468, 2294-2318 (2012).

${ }^{12}$ E. Voyagaki, I. Psycharis, and G. Mylonakis, "Complex resonse of a rocking block to a fullcycle pulse," Journal of Engineering Mechanics 140, 04014024 (2014).

${ }^{13}$ P. Brzeski, T. Kapitaniak, and P. Perlikowski, "The use of tuned mass absorber to prevent overturning of the rigid block during earthquake," International Journal of Structural Stability and Dynamics 16, 1550075 (2016). 
${ }^{14}$ S. Hogan, "The many steady state responses of a rigid block under harmonic forcing," Earthquake Engineering \& Structural Dynamics 19, 1057-1071 (1990).

${ }^{15} \mathrm{~W}$. Tso and C. Wong, "Steady state rocking response of rigid blocks part 1: Analysis," Earthquake engineering \& structural dynamics 18, 89-106 (1989).

${ }^{16} \mathrm{C}$. Wong and W. Tso, “Steady state rocking response of rigid blocks part 2: Experiment," Earthquake engineering \& structural dynamics 18, 107-120 (1989).

${ }^{17}$ S. Lenci and G. Rega, "Heteroclinic bifurcations and optimal control in the nonlinear rocking dynamics of generic and slender rigid blocks," International Journal of Bifurcation and Chaos 15, 1901-1918 (2005).

${ }^{18}$ S. Lenci and G. Rega, "A dynamical systems approach to the overturning of rocking blocks," Chaos, Solitons \& Fractals 28, 527-542 (2006).

${ }^{19}$ A. Kounadis, "Parametric study in rocking instability of a rigid block under harmonic ground pulse: a unified approach," Soil Dynamics and Earthquake Engineering 45, 125-143 (2013).

${ }^{20}$ E. Pavlovskaia, J. Ing, M. Wiercigroch, and S. Banerjee, "Complex dynamics of bilinear oscillator close to grazing," Internat. J. of Bif. and Chaos 20, 3801-3817 (2010).

${ }^{21}$ Y. Liu and J. Páez Chávez, "Controlling coexisting attractors of an impacting system via linear augmentation," Physica D 348, 1-11 (2017).

${ }^{22} \mathrm{~B}$. Guo and Y. Liu, "Three-dimensional map for a piecewise-linear capsule system with bidirectional drifts," Physica D 399, 95-107 (2019).

${ }^{23}$ M. di Bernardo, C. Budd, A. Champneys, and P. Kowalczyk, Piecewise-smooth dynamical systems. Theory and Applications, Applied Mathematical Sciences, Vol. 163 (Springer-Verlag, New York, 2004).

${ }^{24}$ P. Piiroinen and Y. Kuznetsov, "An event-driven method to simulate Filippov systems with accurate computing of sliding motions," ACM Trans. Math. Software 34, 24 (2008).

${ }^{25}$ B. Krauskopf, H. Osinga, and J. Galán-Vioque, eds., Numerical Continuation Methods for Dynamical Systems, Understanding Complex Systems (Springer-Verlag, Netherlands, 2007).

${ }^{26}$ F. Dercole and Y. Kuznetsov, "SlideCont: An Auto97 Driver for Bifurcation Analysis of Filippov Systems," ACM Trans. Math. Software 31, 95-119 (2005).

${ }^{27}$ P. Thota and H. Dankowicz, "TC-HAT: A Novel Toolbox for the Continuation of Periodic Trajectories in Hybrid Dynamical Systems," SIAM J. Appl. Dyn. Sys. 7, 1283-1322 (2008).

${ }^{28}$ H. Dankowicz and F. Schilder, Recipes for continuation, Computational Science and Engineering (SIAM, Philadelphia, 2013). 
${ }^{29}$ Y. Liu and J. Páez Chávez, "Controlling multistability in a vibro-impact capsule system,” Nonlinear Dynamics 88, 1289-1304 (2017).

${ }^{30}$ Y. Liu, J. Páez Chávez, E. Pavlovskaia, and M. Wiercigroch, "Analysis and control of the dynamical response of a higher order drifting oscillator,” Proc. R. Soc. A 474, 20170500 (2018).

${ }^{31}$ P. Menck, J. Heitzig, N. Marwan, and J. Kurths, "How basin stability complements the linearstability paradigm," Nature Physics 9, 89-92 (2013).

${ }^{32}$ P. Schultz, P. Menck, J. Heitzig, and J. Kurths, "Potentials and limits to basin stability estimation,” New Journal of Physics 19, 023005 (2017).

${ }^{33}$ P. Brzeski, P. Belardinelli, S. Lenci, and P. Perlikowski, "Revealing compactness of basins of attraction of multi-dof dynamical systems," Mechanical Systems and Signal Processing 111, 348-361 (2018).

${ }^{34}$ P. Belardinelli, B. Sajadi, S. Lenci, and F. Alijani, "Global dynamics and integrity of a microplate pressure sensor," Communications in Nonlinear Science and Numerical Simulation 69, 432-444 (2019).

${ }^{35}$ A. Chandrashekar, P. Belardinelli, U. Staufer, and F. Alijani, "Robustness of attractors in tapping mode atomic force microscopy," Nonlinear Dynamics 97, 1137-1158 (2019).

${ }^{36}$ A. Chudzik, P. Perlikowski, A. Stefanski, and T. Kapitaniak, "Multistability and rare attractors in van der pol-duffing oscillator," International Journal of Bifurcation and Chaos 21, 1907-1912 (2011).

${ }^{37}$ S. Brezetskyi, D. Dudkowski, and T. Kapitaniak, "Rare and hidden attractors in van der polduffing oscillators,” The European Physical Journal Special Topics 224, 1459-1467 (2015).

${ }^{38}$ D. Dudkowski, A. Prasad, and T. Kapitaniak, "Perpetual points and hidden attractors in dynamical systems," Physics Letters A 379, 2591-2596 (2015).

${ }^{39}$ A. Klokov and M. Zakrzhevsky, "Parametrically excited pendulum systems with several equilibrium positions: Bifurcation analysis and rare attractors," International Journal of Bifurcation and Chaos 21, 2825-2836 (2011).

${ }^{40}$ P. Brzeski, M. Lazarek, T. Kapitaniak, J. Kurths, and P. Perlikowski, "Basin stability approach for quantifying responses of multistable systems with parameters mismatch," Meccanica 51, 2713-2726 (2016).

${ }^{41}$ P. Brzeski, J. Wojewoda, T. Kapitaniak, J. Kurths, and P. Perlikowski, “Sample-based approach can outperform the classical dynamical analysis-experimental confirmation of the basin stability method," Scientific reports 7, 1-10 (2017). 
${ }^{42}$ P. Brzeski and P. Perlikowski, "Sample-based methods of analysis for multistable dynamical systems," Archives of Computational Methods in Engineering 26, 1515-1545 (2019).

${ }^{43}$ S. Yanchuk and T. Kapitaniak, "Manifestation of riddling in the presence of a small parameter mismatch between coupled systems," Physical Review E 68, 017202 (2003).

${ }^{44}$ M. Lazarek, P. Brzeski, W. Solecki, and P. Perlikowski, "Detection and classification of solutions for systems interacting by soft impacts with sample-based method," International Journal of Bifurcation and Chaos 30, 2050079 (2020). 


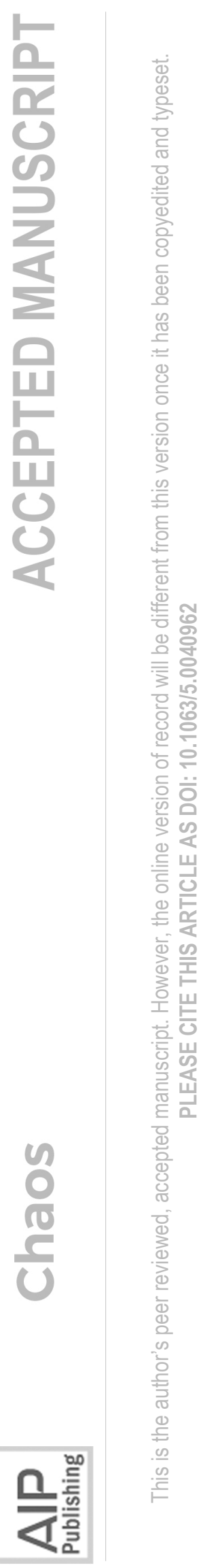




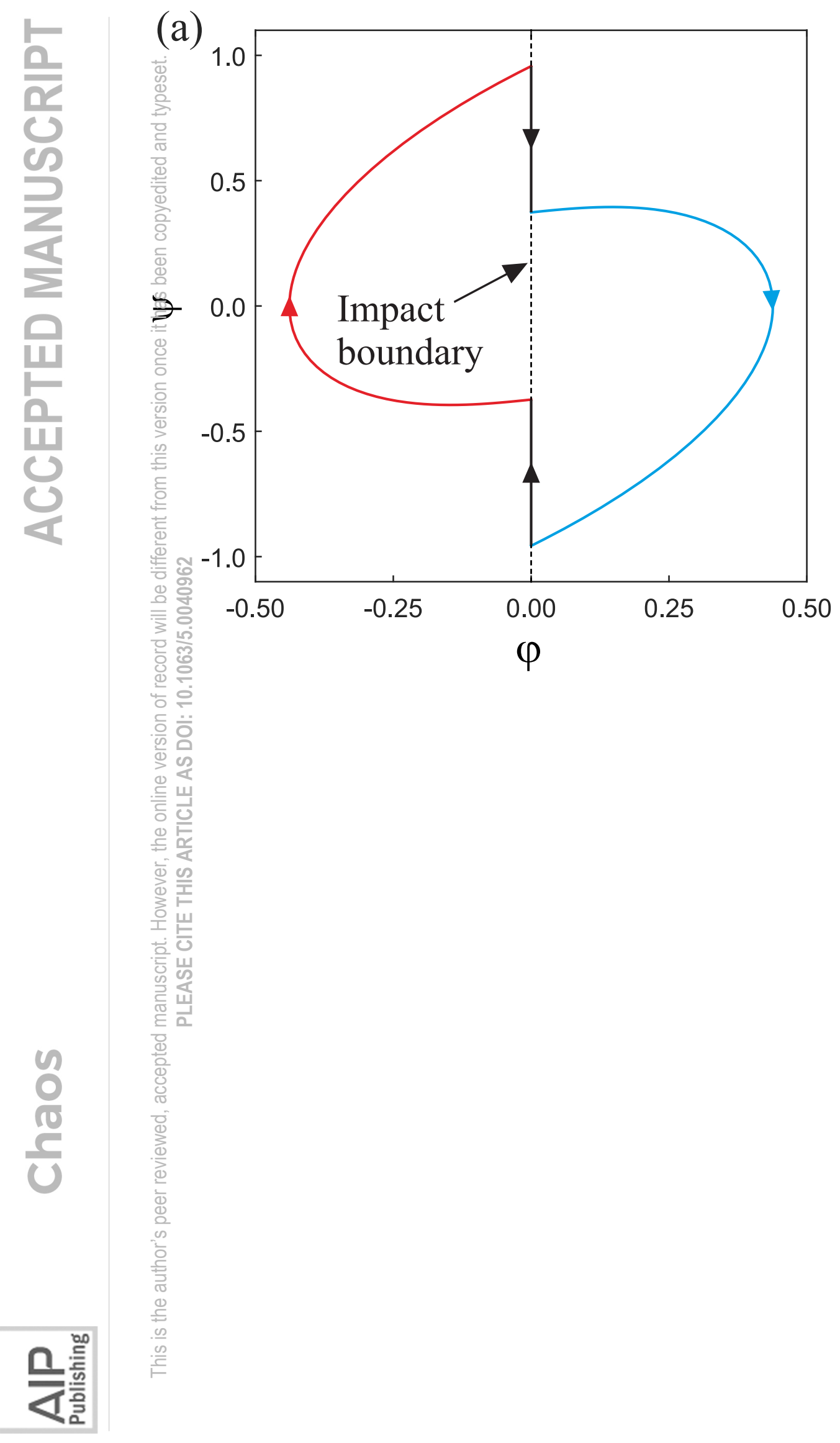

(b)

Impact boundary 
(a)
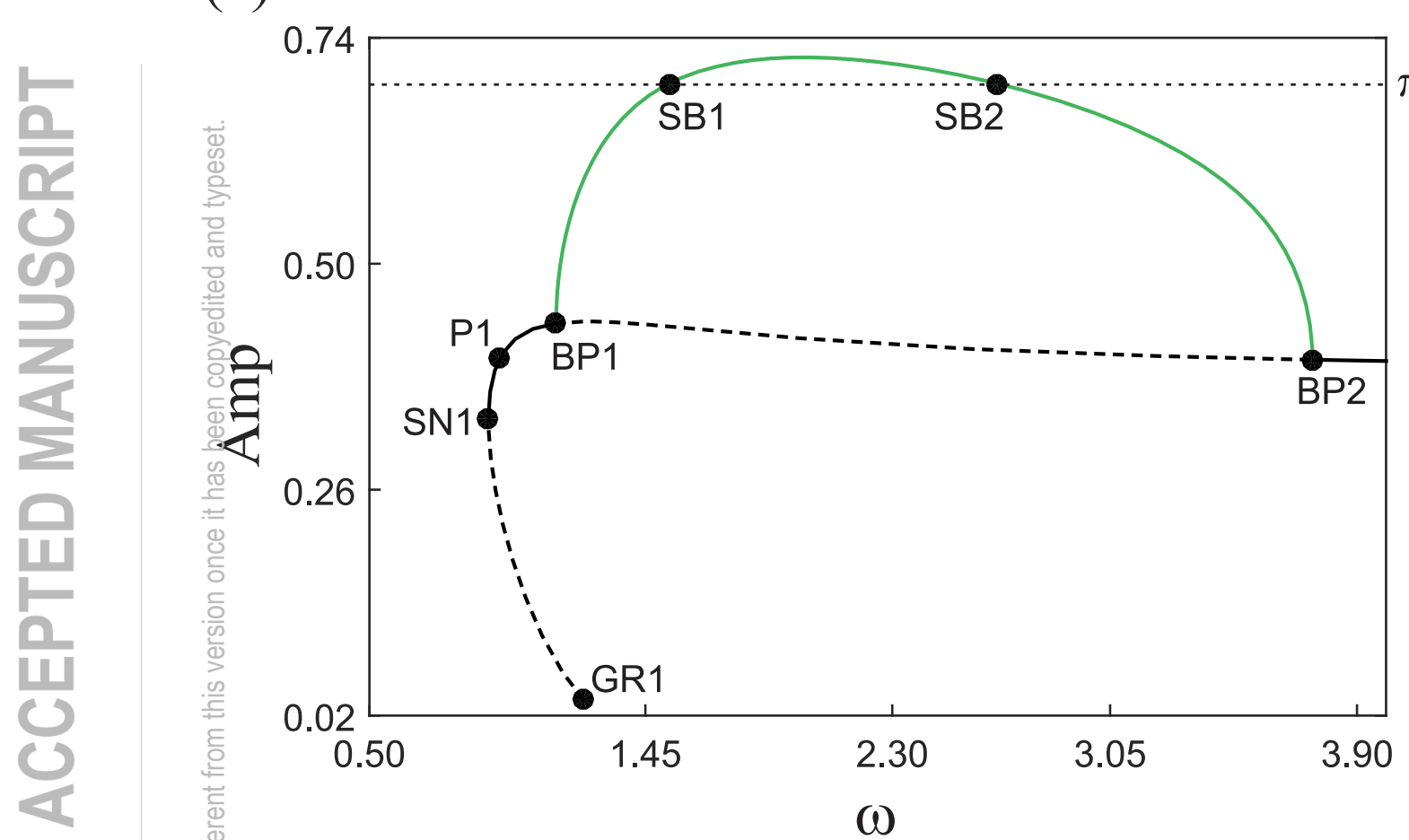

(6)

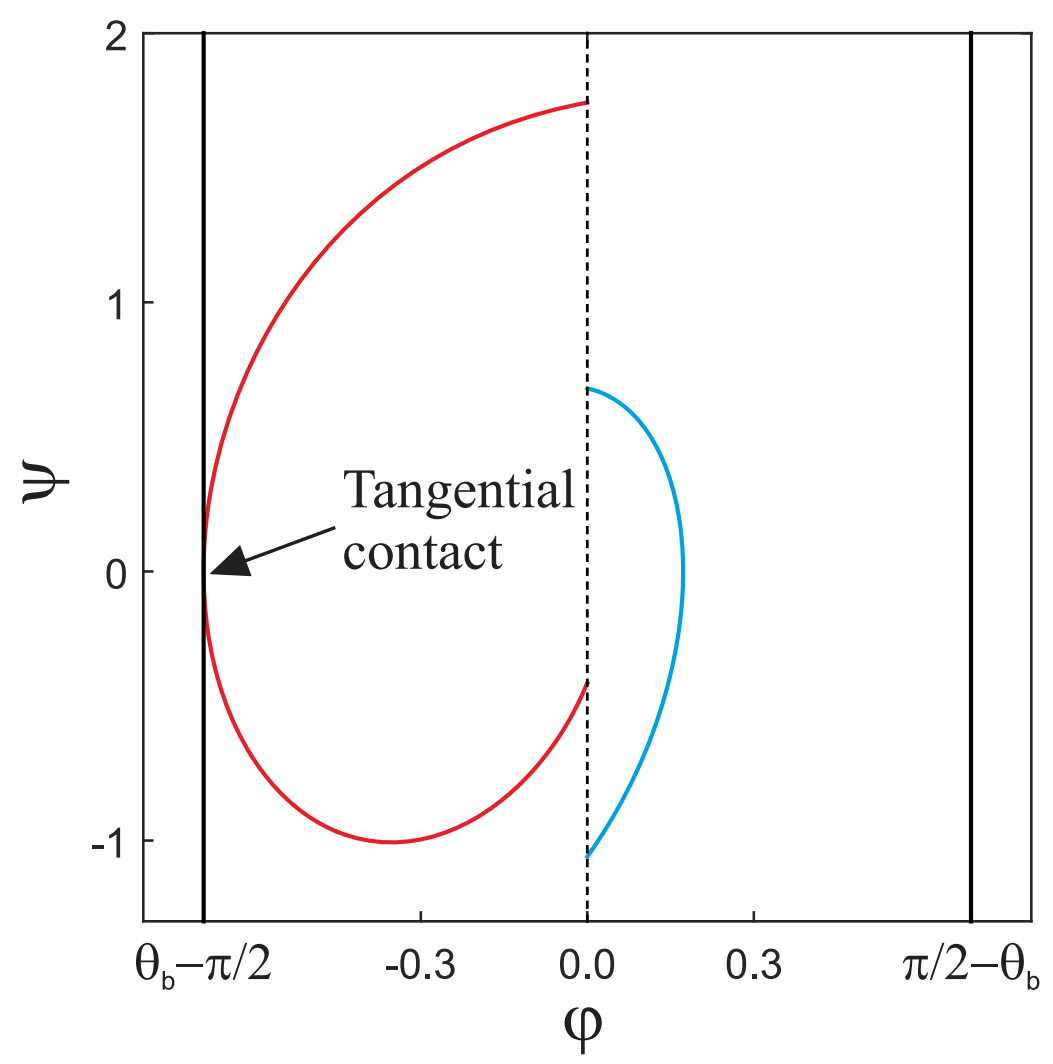

(b) $\pi / 2-\theta_{b}$

(d)

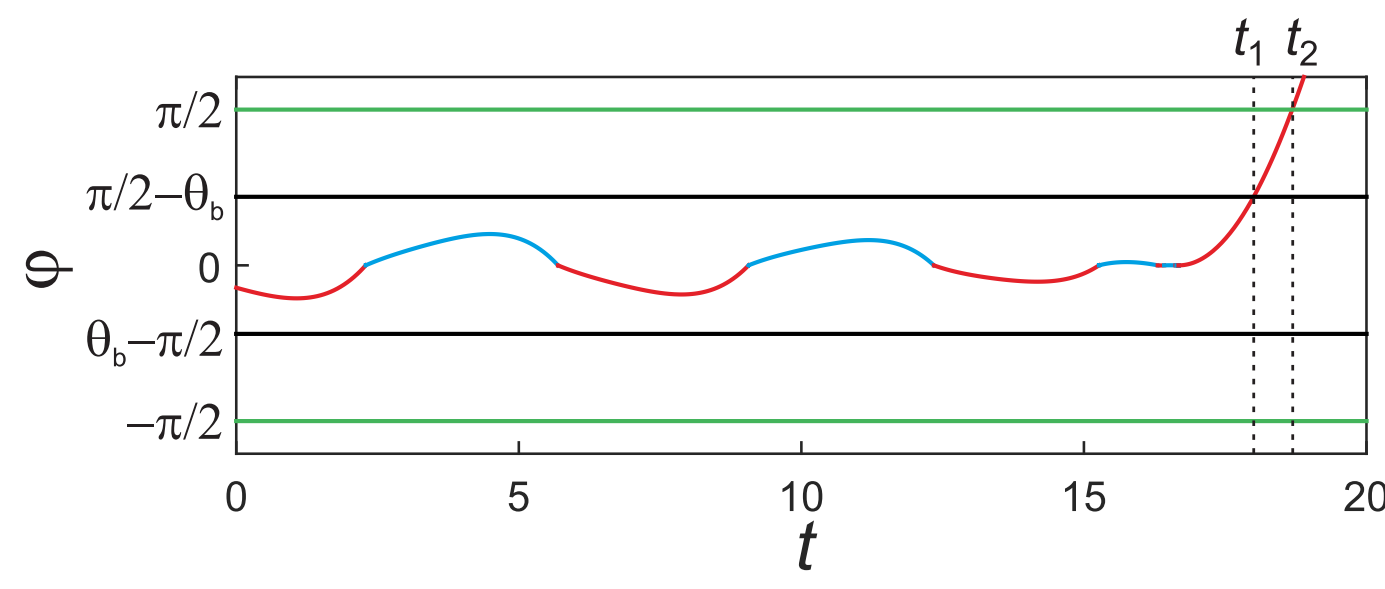


(a)
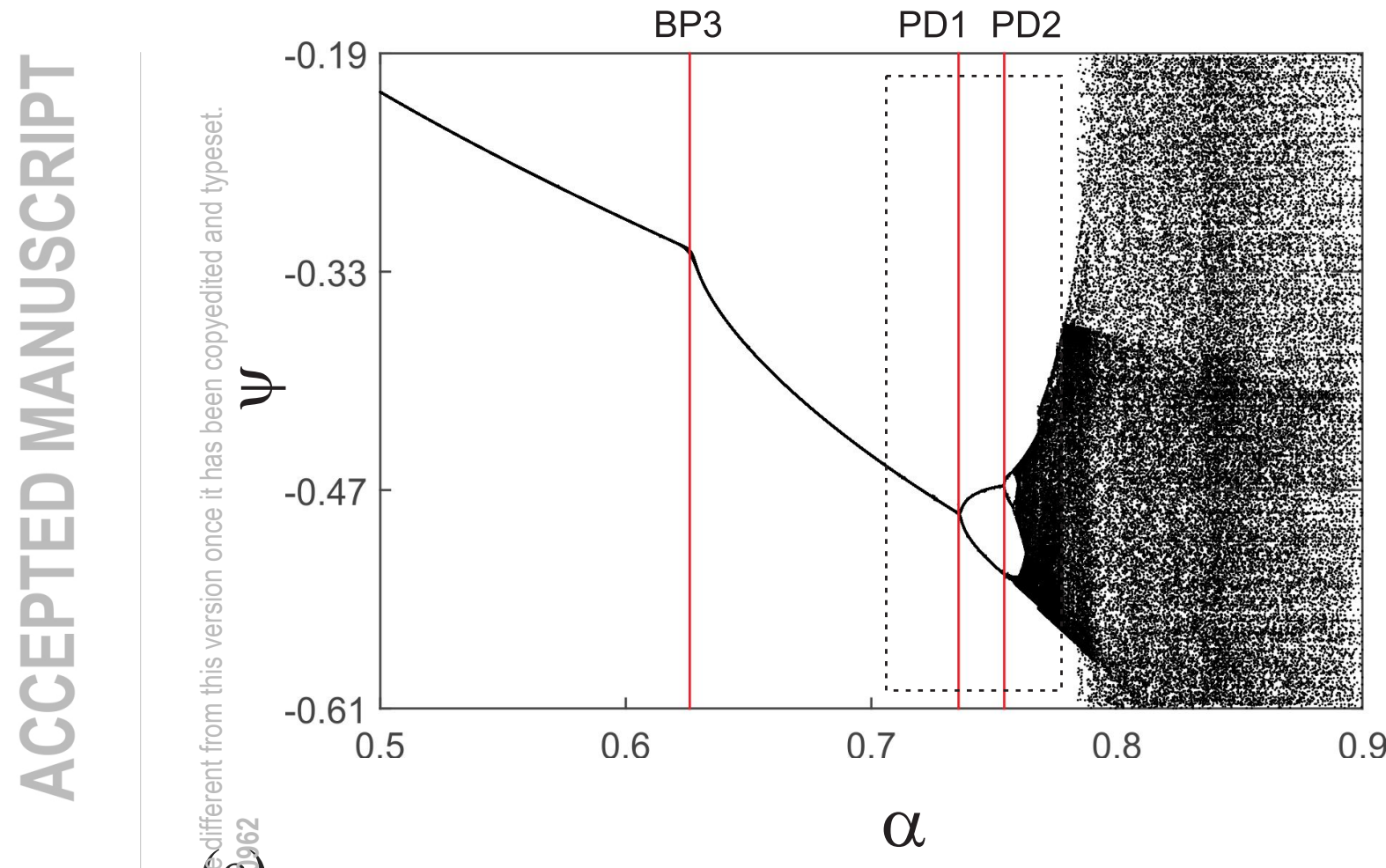

(c)

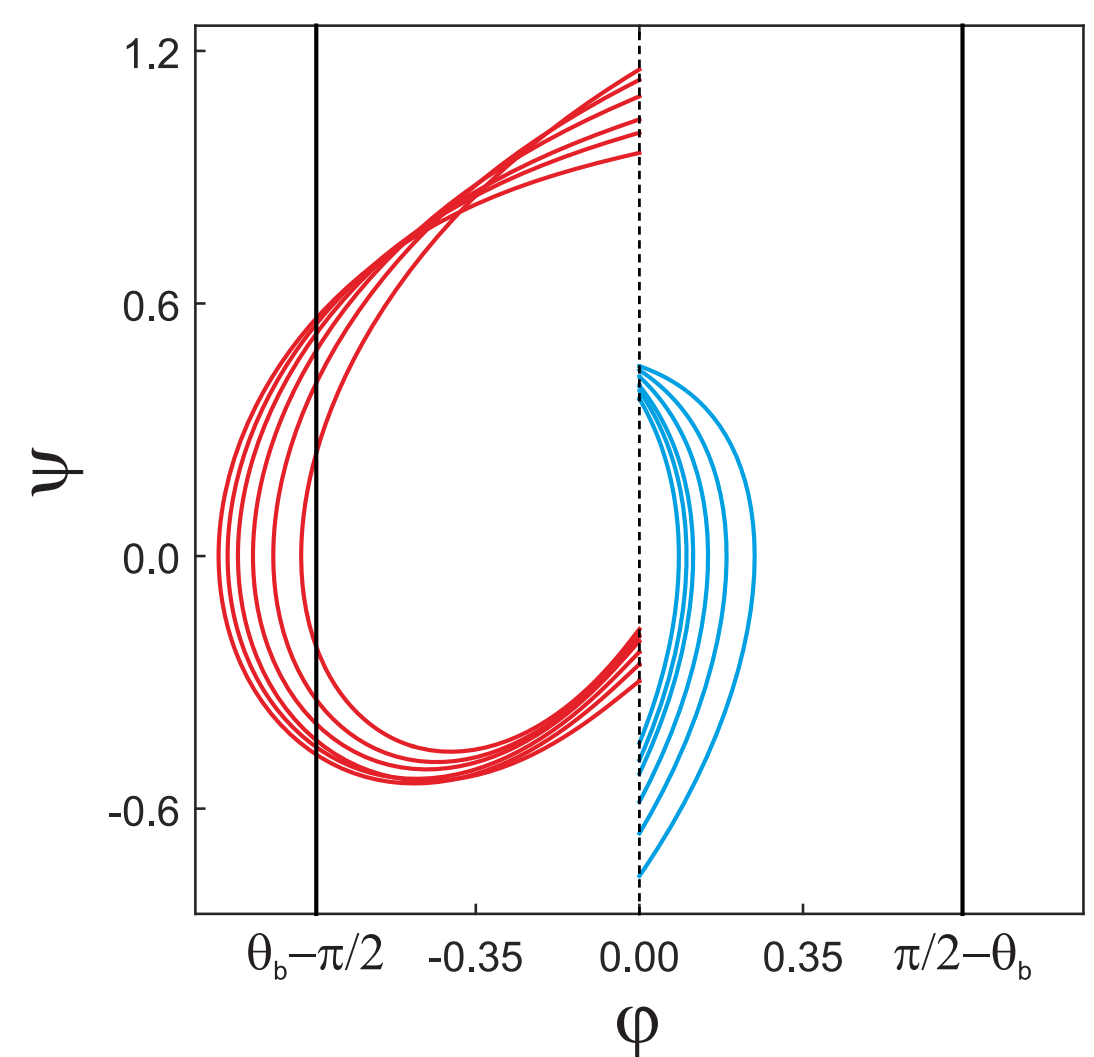

(e)

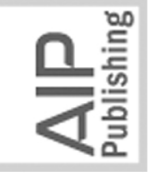

(b)

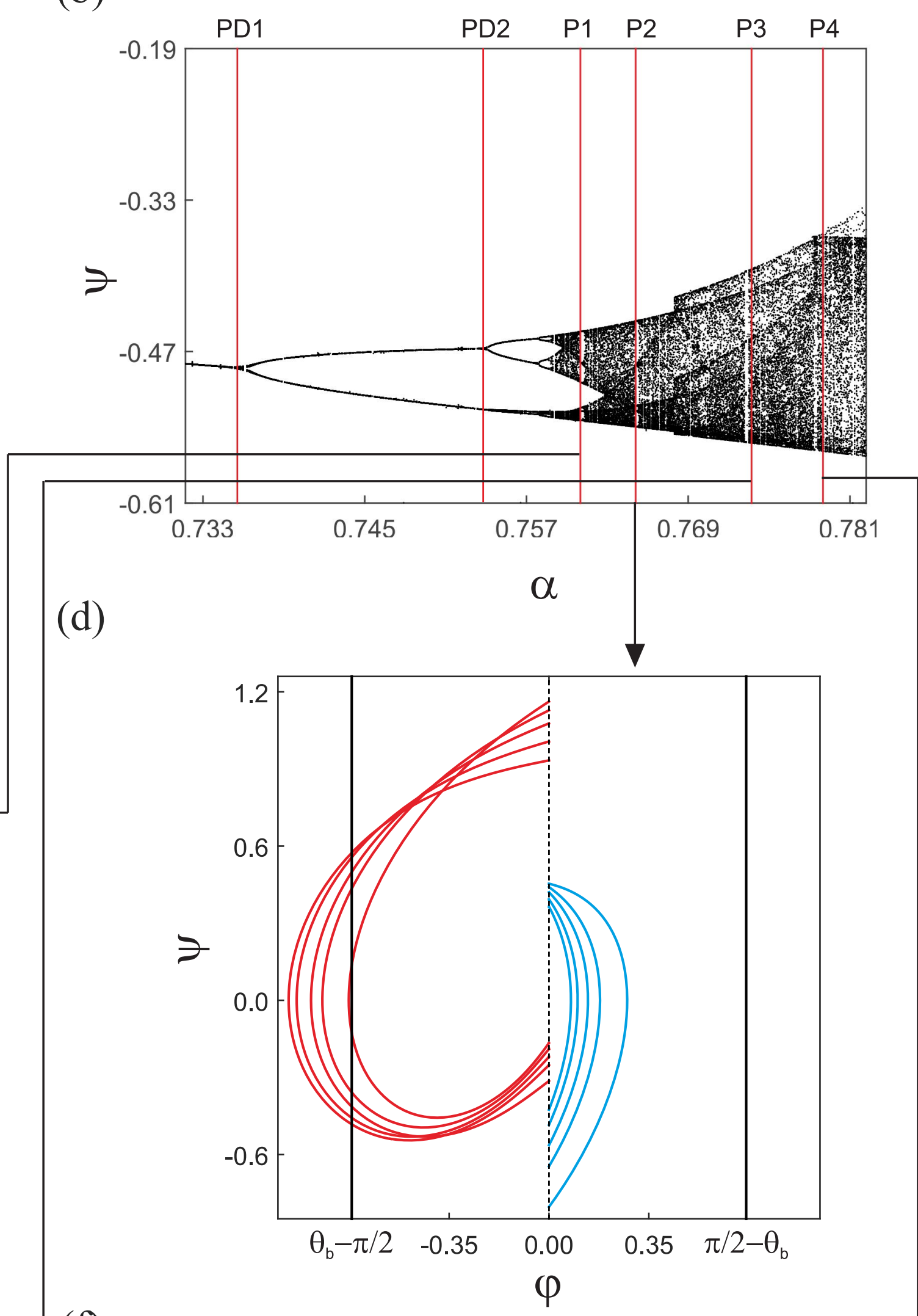

(f)

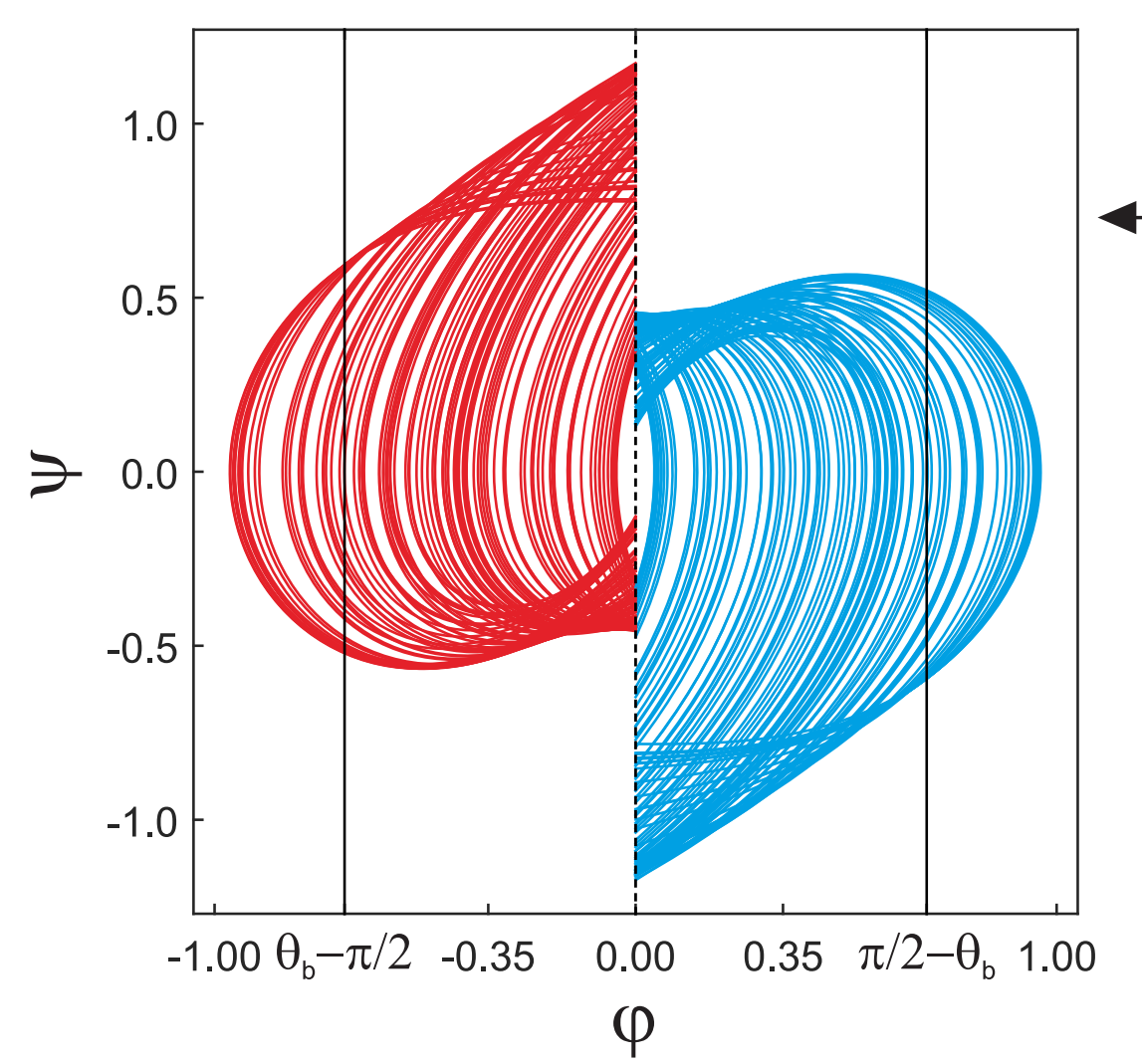


(a)
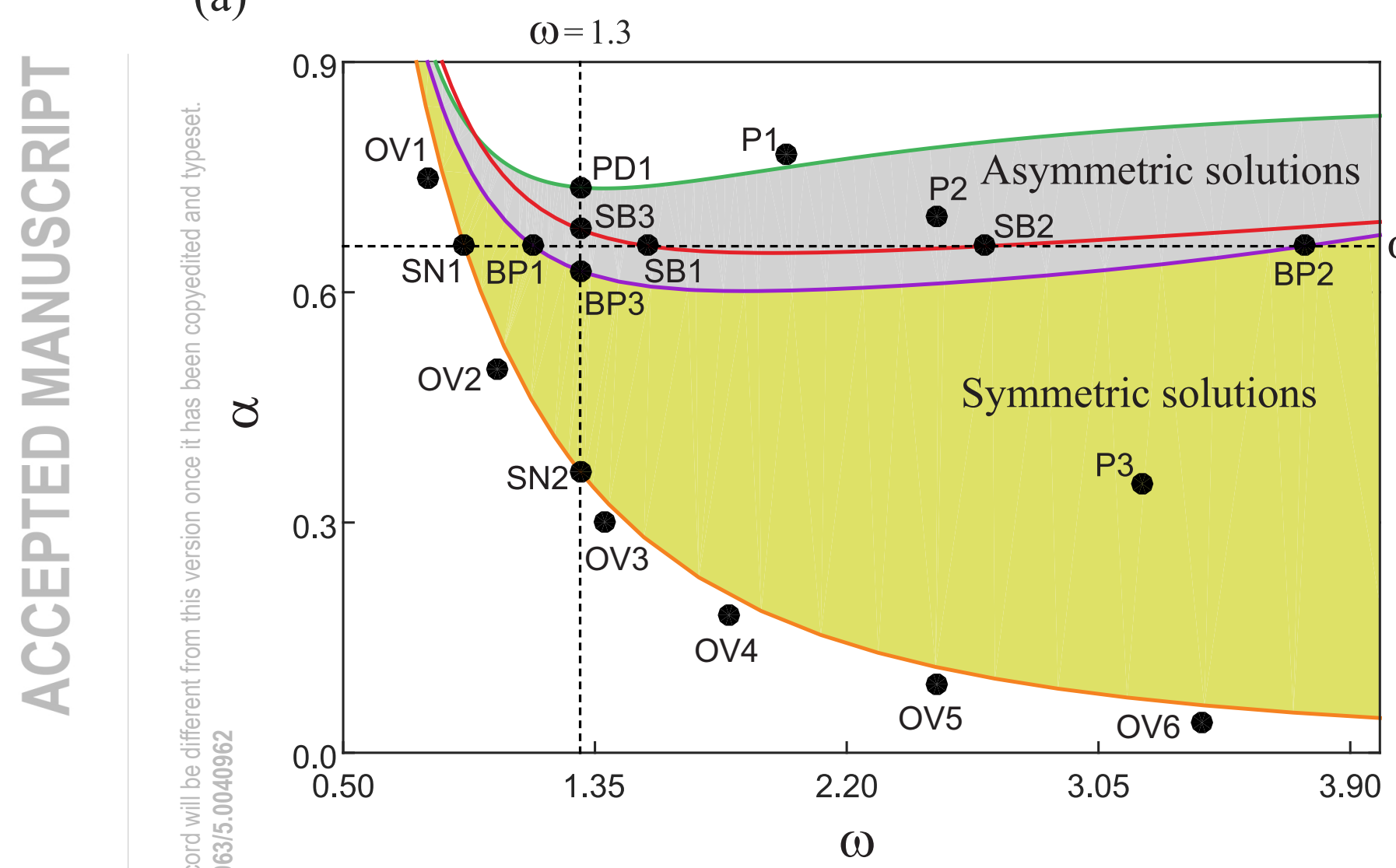

(⿳) (e)

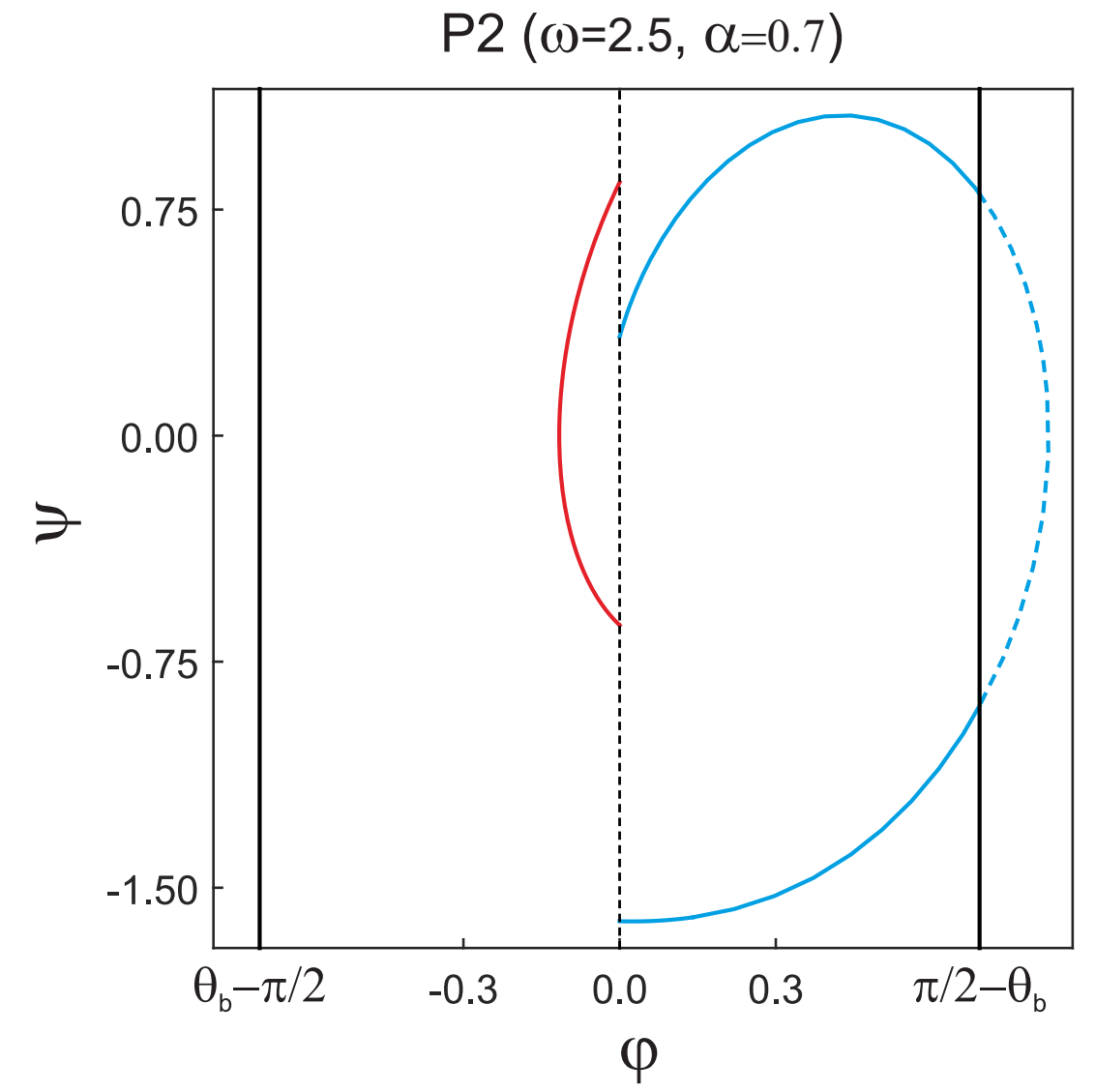

(e)

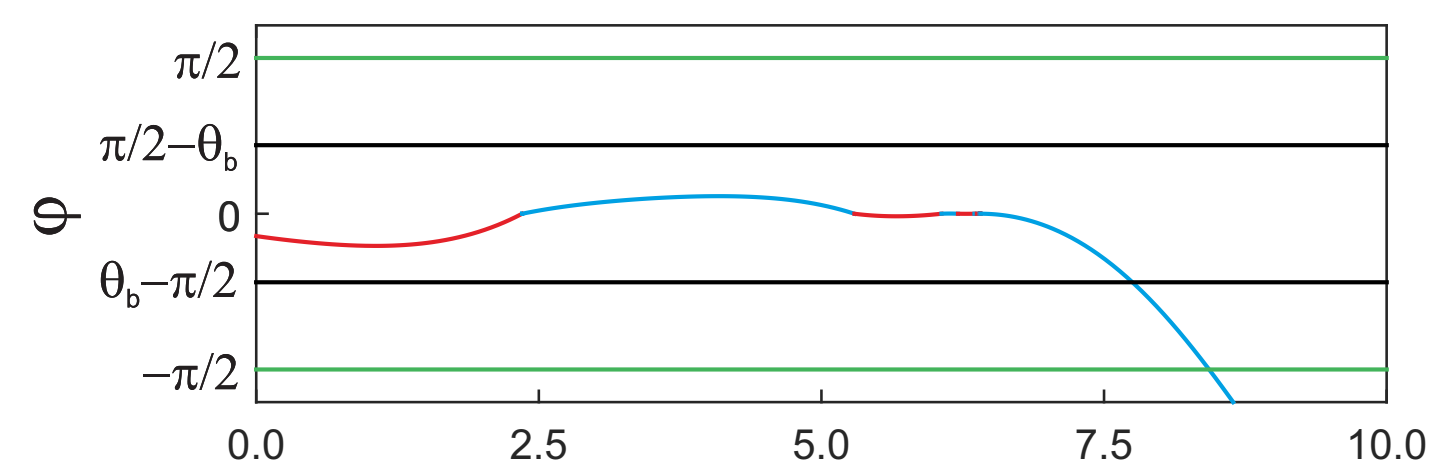

OV2 $(\omega=1.02, \alpha=0.5)$

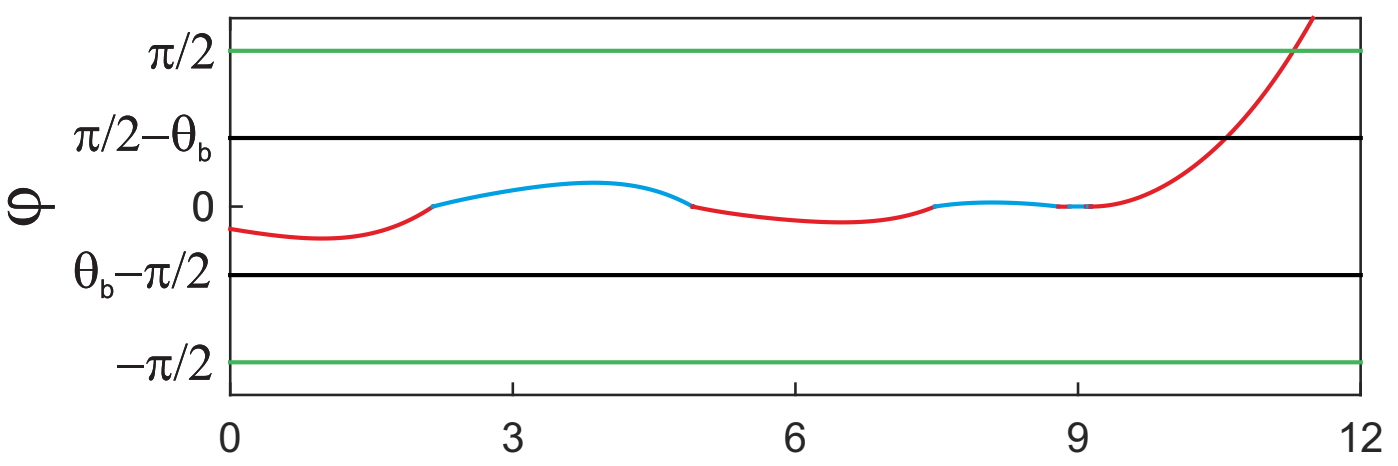

OV3 $(\omega=1.38, \alpha=0.3)$

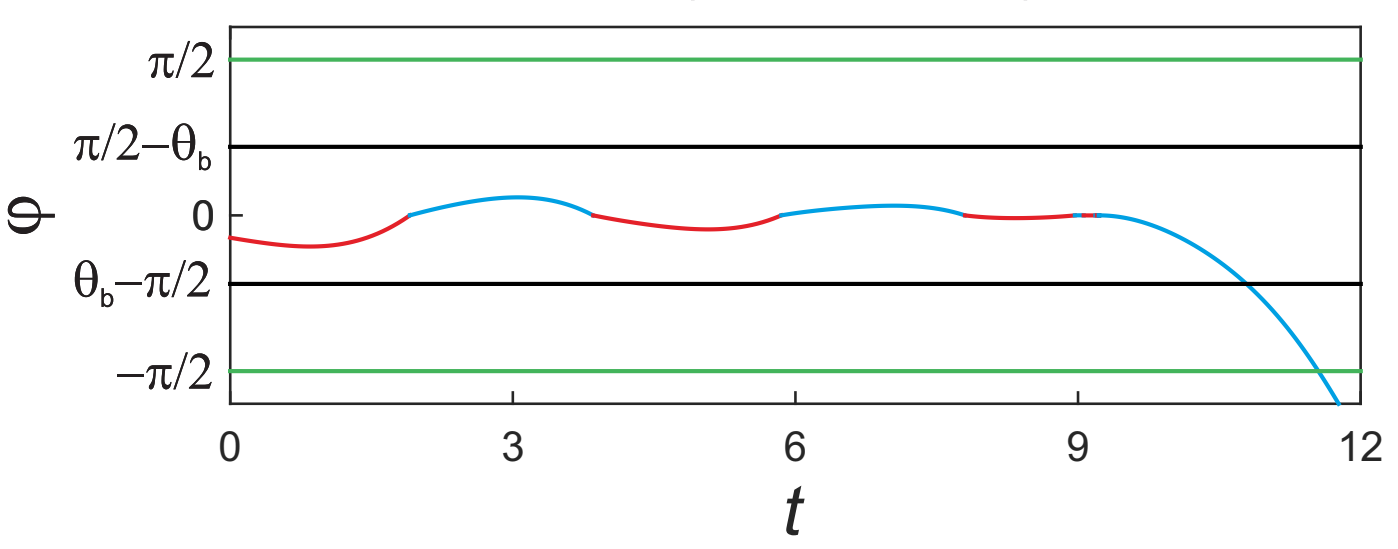

(b)

$\mathrm{P} 1(\omega=2, \alpha=0.78)$

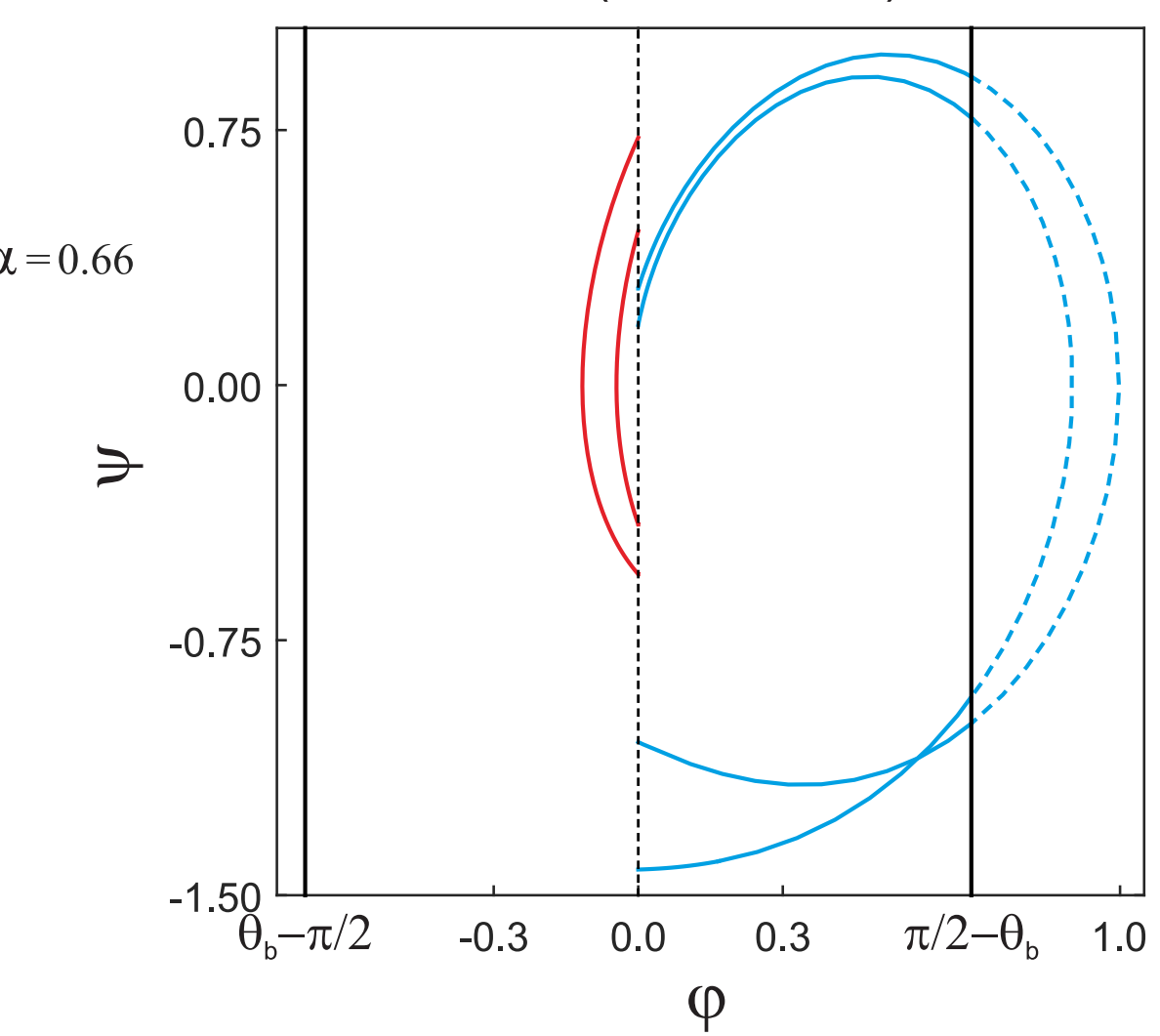

(d)

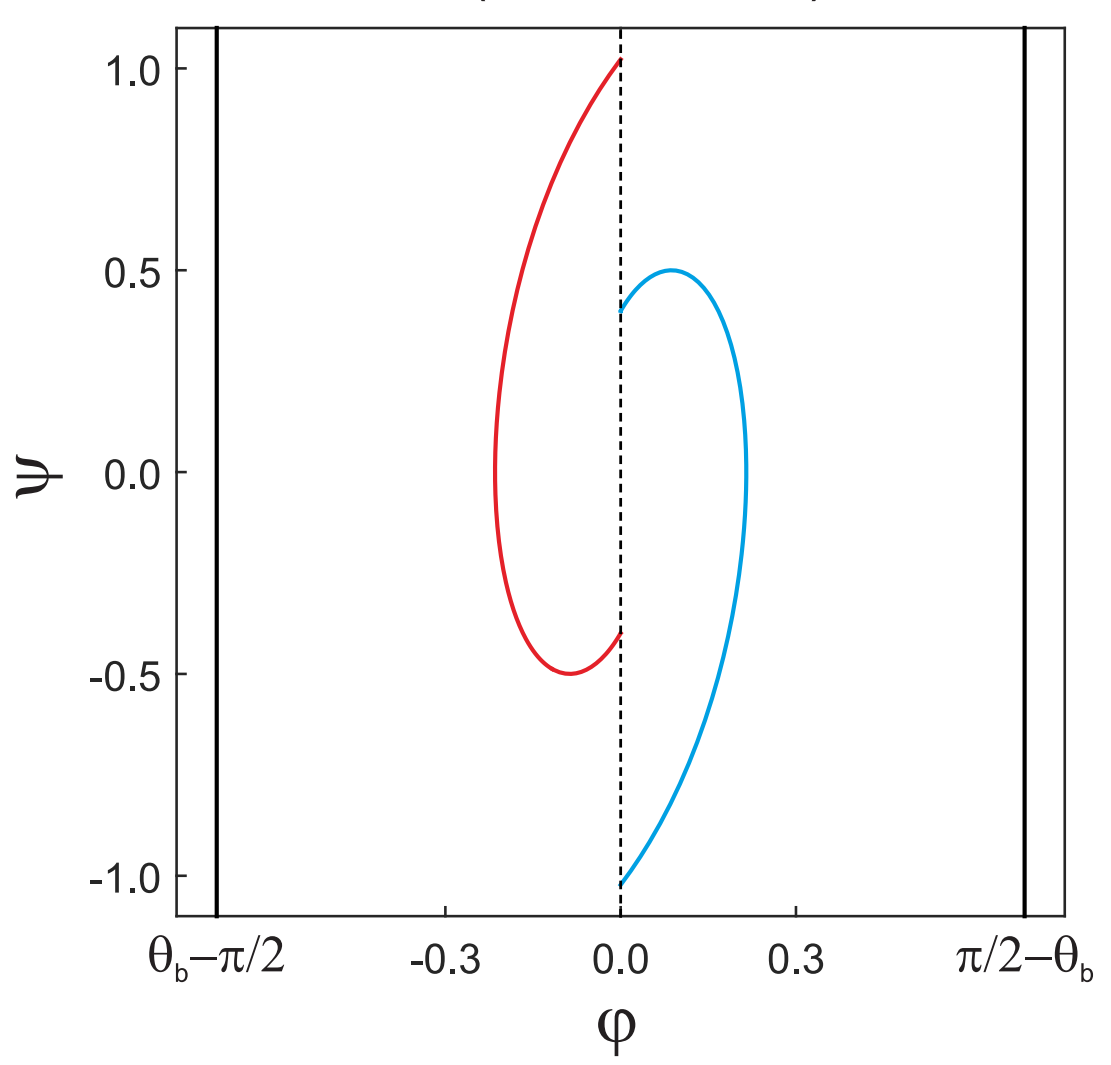

OV4 ( $\omega=1.8, \alpha=0.18)$

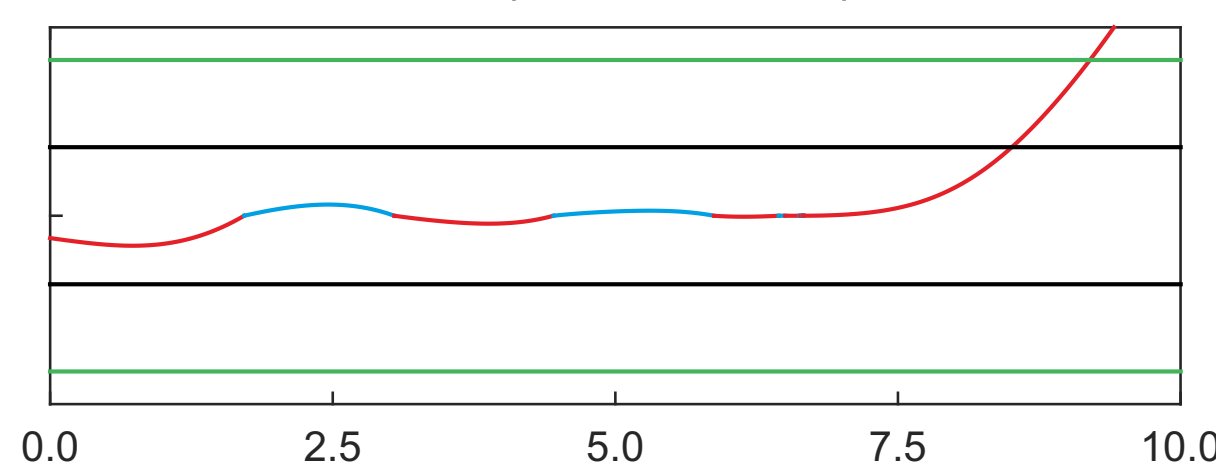

OV5 ( $\omega=2.5, \alpha=0.09)$

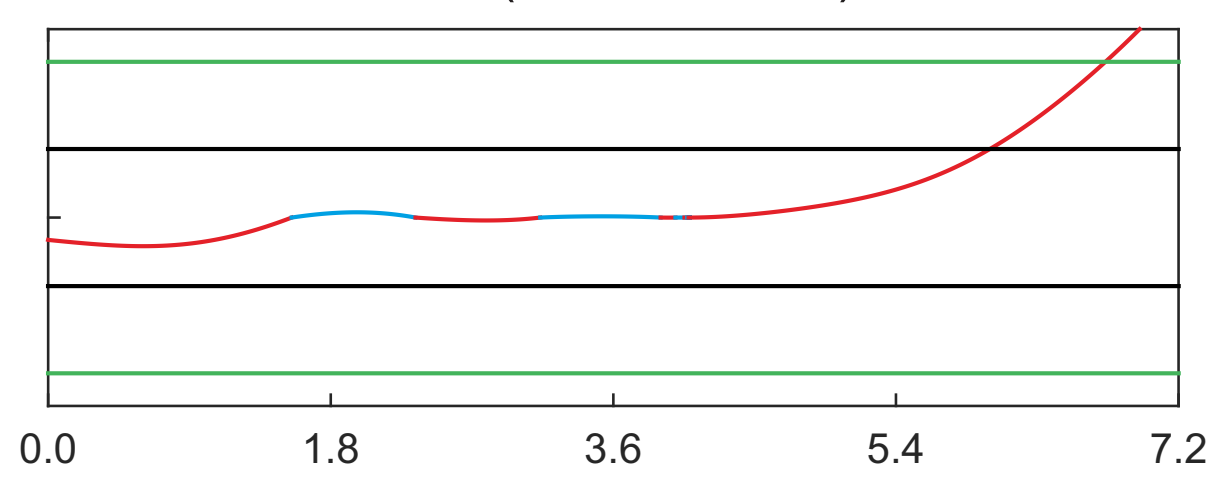

OV6 $(\omega=3.4, \alpha=0.04)$

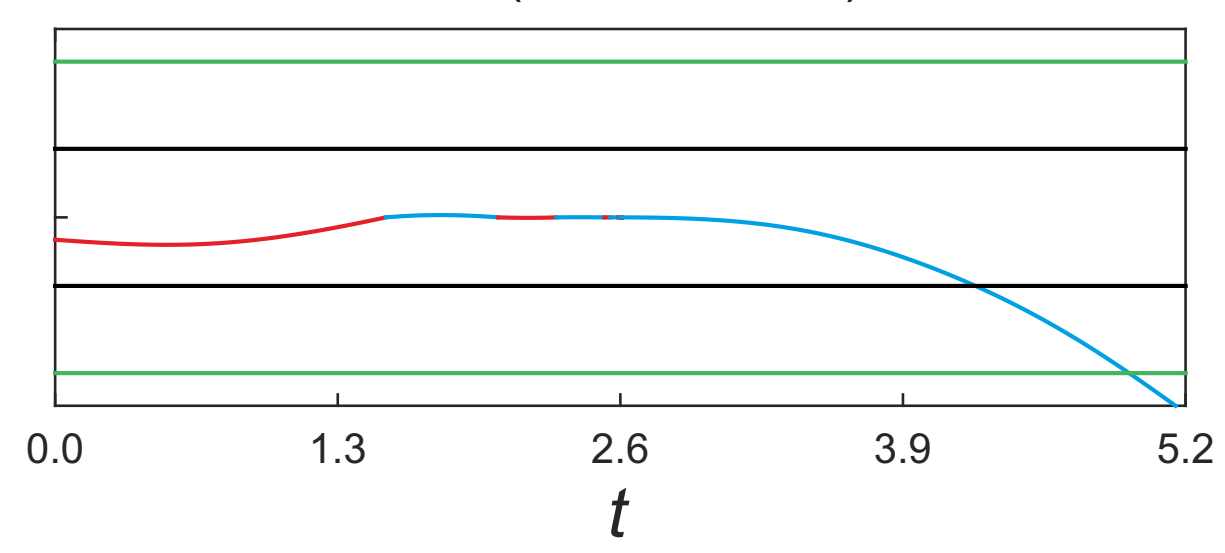


(a)

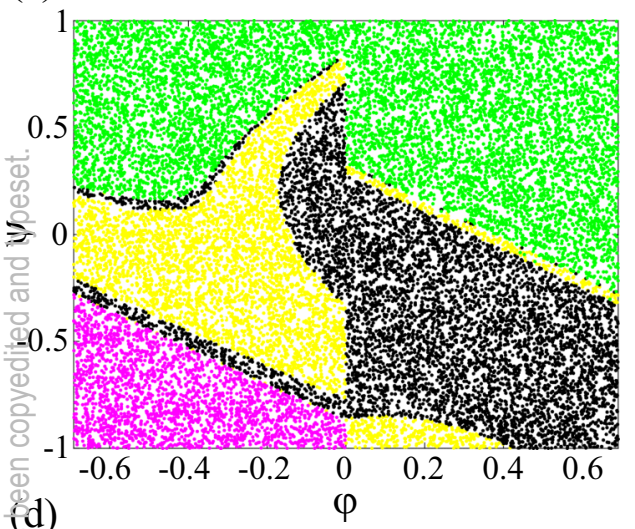

(d)

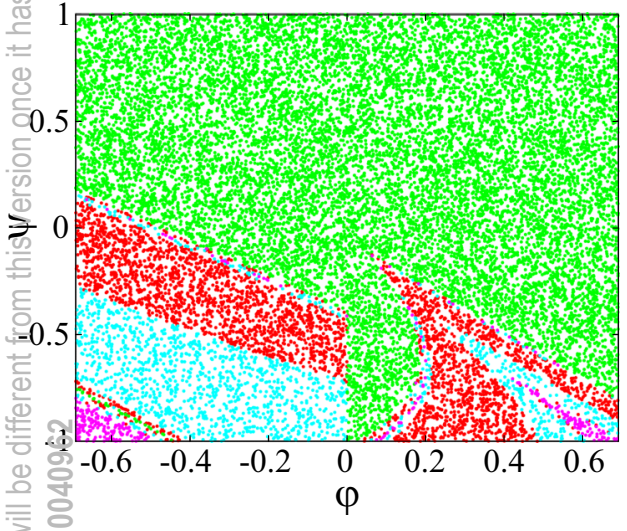

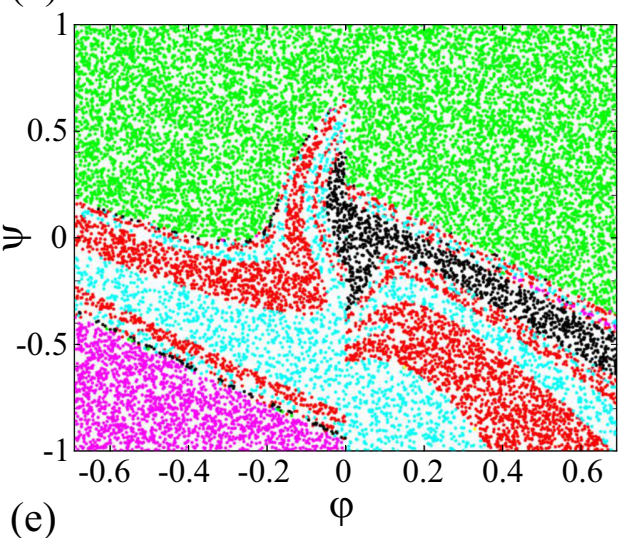

(e)

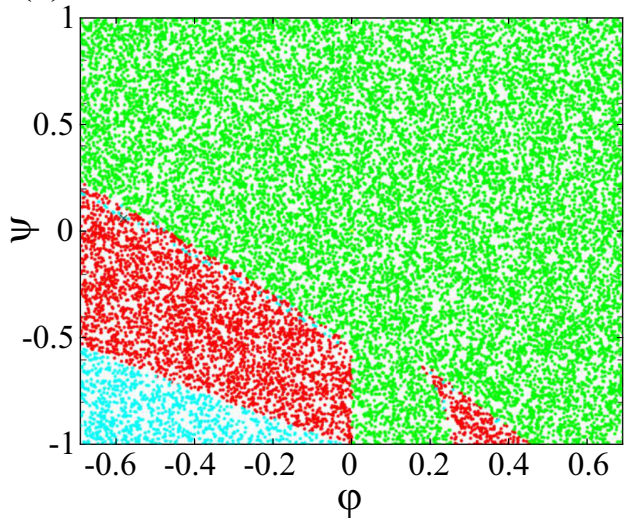

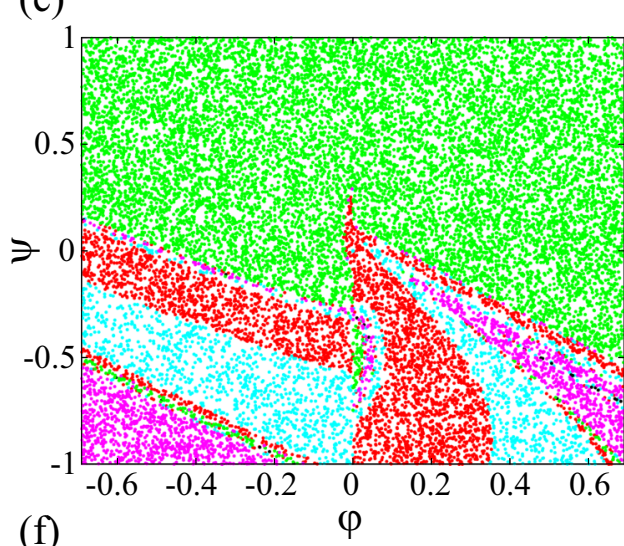

(f)

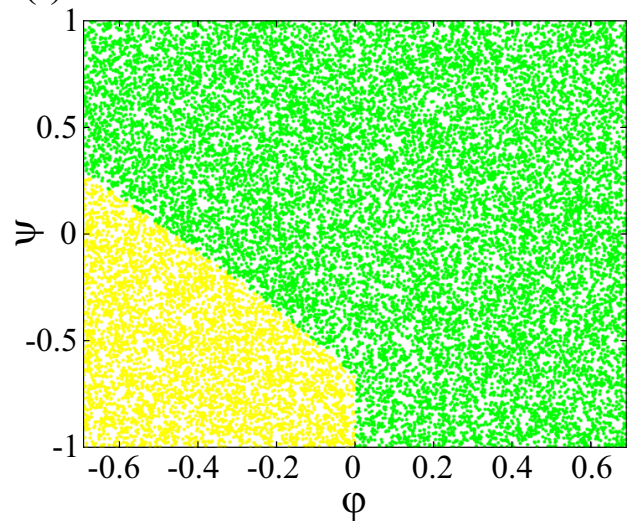

- Period 1 asym + Period $1 \cdot$ Overturn $+\pi / 2 \cdot$ Chaos and period $>2$

- Period 1 asym - $\quad \times$ Period 2 - Overturn $-\pi / 2 \bullet$ Equlibrium 

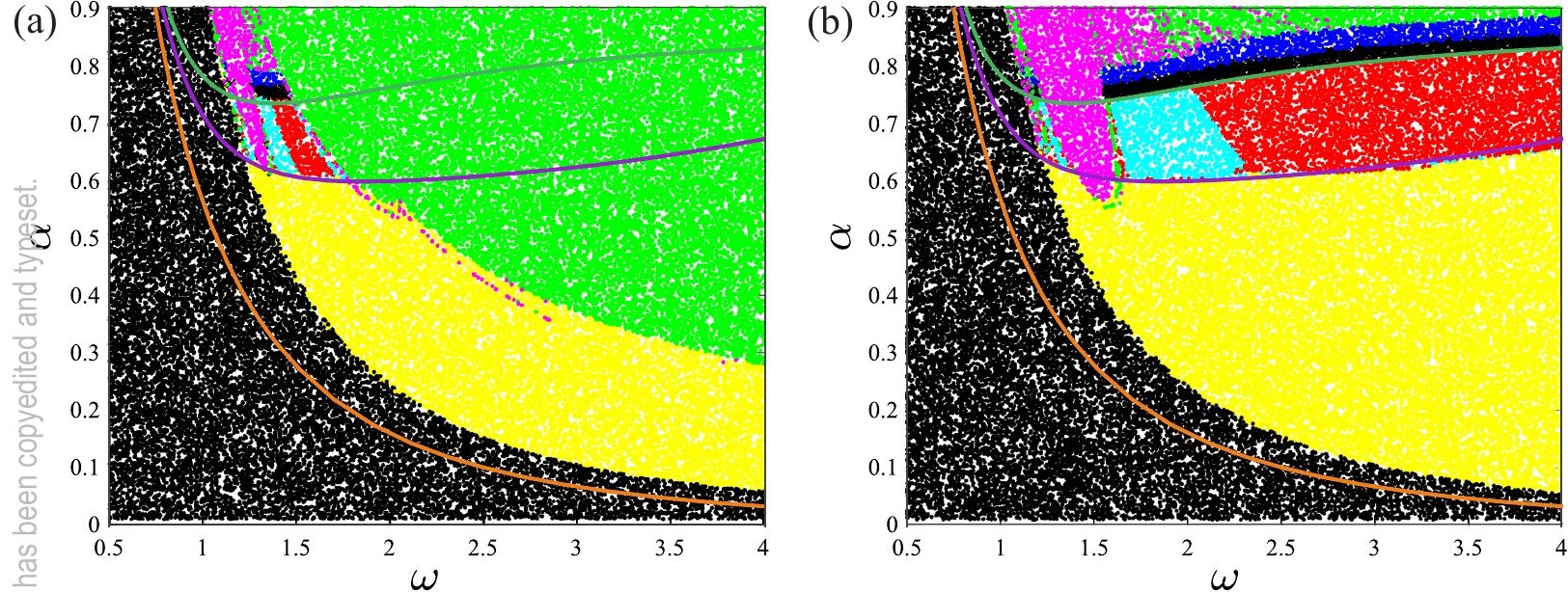

- Period 1 asym + Period 1 - Overturn $+\pi / 2$ - Chaos and period $>2$

- Period 1 asym - $\times$ Period 2 - Overturn $-\pi / 2 \bullet$ Equlibrium 


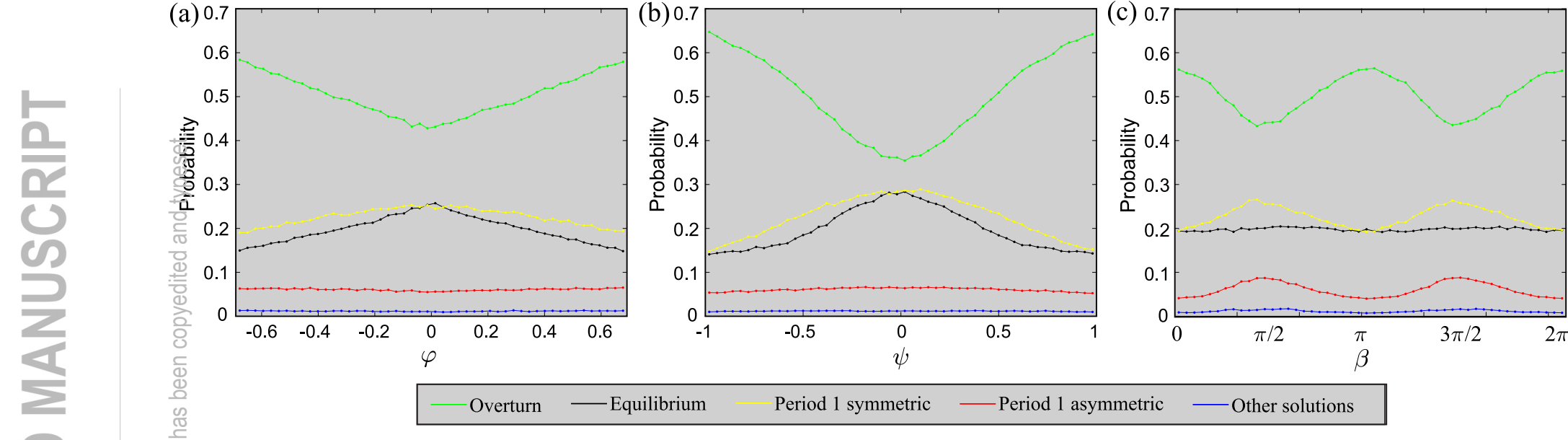

